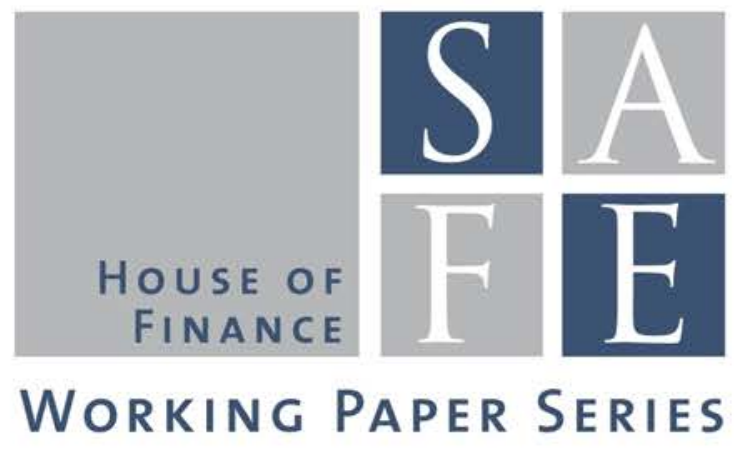

Nicole Branger - Paulo Rodrigues - Christian Schlag

\title{
Level and Slope of Volatility Smiles in Long-Run Risk Models
}

SAFE Working Paper No. 186

SAFE I Sustainable Architecture for Finance in Europe A cooperation of the Center for Financial Studies and Goethe University Frankfurt 


\section{Non-Technical Summary}

Understanding the fundamental economic risks that drive asset prices is a central research question in finance. Derivatives markets, in conjunction with their underlying stock markets, provide us with a rich set of data to study this topic. It is well established that there are two main types of risks driving asset prices. First, there is diffusive volatility, which measures the amount of uncertainty generated by 'usual' movements in economic fundamentals In asset pricing models, this risk is mostly represented by normally distributed innovations. Second, there are large, and infrequent shocks which generate rapid (and mostly adverse) changes in these fundamentals. These are usually represented by jumps. Due to the sheer size of the movements in state variables (and consequently in prices) conditional on the occurrence of a jump, this type of risk is usually much more dramatic than that generated by diffusive factors, it is harder to hedge and thus has much severer implications for wealth dynamics and risk premia.

In many models it is assumed that the risk of such a jump occurring is the higher, the higher the current level of diffusive volatility, which in these models implies a perfect correlation of both risk types. We show in this paper that a simple separation of volatility and jump risk suffices for the model to match two basic and easily observable characteristics of the implied volatility (IV) smile for S\&P 500 index options, namely its level and its slope. This improvement in explanatory power is achieved in addition to the matching of basic asset pricing moments like the equity premium, the volatility of the equity premium, the variance premium, and excess return predictability by the price-dividend ratio and the variance risk premium. The important feature of our model from an economic standpoint is therefore that the probability of a large shock is not perfectly correlated with the 'usual' uncertainty represented by diffusive variance.

Roughly speaking, the level of the IV smile represents the amount of volatility, while the slope measures jump risk. In contrast to the hypotheses implied by standard models, the correlation between level and slope is far from perfectly positive, and it is even negative with a value around -0.3 for our sample for the S\&P 500 index from 1996 to 2015, and even in a general nonlinear sense, the goodness of fit for slope as a function of level is rather low. Our model with separate processes for volatility and jump risk is able to explain these key properties of the data, while standard models are clearly not.

The weak link between level and slope is not just of technical interest in the context of the asset pricing model discussed in our paper, but the two risk types related to level and slope are also fundamentally different economically. Overall, we find that both level and slope are related to macro variables, but the degree to which level can be explained by a collection of such variables is much higher than for slope.

To sum up, we propose a simple modification and extension of existing asset pricing models, which goes a long way in explaining stylized facts from options markets. The results allow deeper insights into the different types of risks priced on asset markets and their comovement, which is important for applications like asset allocation and risk management. 


\title{
Level And Slope of Volatility Smiles in Long-Run Risk MODELS
}

\author{
Nicole BRANGER ${ }^{\dagger}$ \\ Finance Center Muenster, University of Muenster, Germany \\ Paulo Rodrigues ${ }^{\ddagger}$ \\ Maastricht University, Maastricht, Netherlands \\ Christian SCHLAG* \\ Research Center SAFE, Goethe University Frankfurt, Germany
}

This version: October 16, 2017

We propose a long-run risk model with stochastic volatility, a time-varying mean reversion level of volatility, and jumps in the state variables. The special feature of our model is that the jump intensity is not affine in the conditional variance but driven by a separate process. We show that this separation of jump risk from volatility risk is needed to match the empirically weak link between the level and the slope of the implied volatility smile for S\&P 500 options.

Keywords Asset pricing, Epstein-Zin preferences, jump risk, stochastic volatility, level and slope of implied volatility smile

\section{JEL Classifications: G12}

\footnotetext{
${ }^{\dagger}$ Universitaetsstrasse 14-16, 48143 Muenster, Germany, e-mail: nicole.branger@wiwi.uni-muenster.de, phone: +49 25183.

¥ Corresponding author. P.O. Box 616, 6200 MD Maastricht, The Netherlands, e-mail: p.rodrigues@maastrichtuniversity.nl, phone: +31 433883633

* Theodor-W.-Adorno-Platz 3, 60323 Frankfurt am Main, Germany, e-mail:schlag@finance.uni-frankfurt.de, phone: +49 69 798-33699. Earlier versions of this paper were presented at the universities of Bonn, Tuebingen, Vienna, at the 12th Symposium on Finance, Banking, and Insurance in Karlsruhe, at the 2012 ESSFM Meetings in Gerzensee, and at the annual meetings of the German Finance Association 2012. We thank all conference and workshop participants for their comments and suggestions. All remaining errors are our responsibility. Christian Schlag gratefully acknowledges financial support by Deutsche Forschungsgemeinschaft (DFG) and research support from SAFE, funded by the State of Hessen initiative for research LOEWE.
} 


\section{Introduction and Motivation}

Understanding the fundamental economic risks that drive asset prices is a central research question in finance. Derivatives markets, in conjunction with their underlying stock markets, provide us with a rich set of data to study this topic. It is well established that time varying variance as well as jump components are important risk factors driving asset prices. For example, in a recent paper Drechsler and Yaron (2011) show that an extension of the long-run risk (LRR) framework of Bansal and Yaron (2004) is able to account for the time variation and return predictability of the variance risk premium. In their analysis Drechsler and Yaron (2011) show that the addition of large infrequent shocks, i.e., jumps, to economic fundamentals allows to explain the existence and the dynamic properties of the variance risk premium. Intuitively, it is shown that economic fundamentals are driven by two types of risks: First, there is diffusive conditional volatility, which measures the amount of uncertainty generated by 'usual' movements in fundamentals and state variables at a certain point in time. This risk is mostly represented by normally distributed innovations. Second, there are large, non-normal, and infrequent shocks which generate rapid (and mostly adverse) changes in fundamentals. Due to the sheer size of the movements in state variables (and consequently in prices) conditional on the occurrence of a jump, this type of risk is usually much more dramatic than that generated by diffusive factors, it is harder to hedge and thus has much severer implications for wealth dynamics and risk premia. A key feature of the model in Drechsler and Yaron (2011), however, is that the jump intensity is an affine function of the conditional variance, which implies a perfect correlation of both risk types.

We show in this paper that a simple separation of variance risk and jump intensity suffices for the model to match two basic and easily observable characteristics of the implied volatility (IV) smile for S\&P 500 index options, namely its level and its slope. This improvement in explanatory power is achieved in addition to the matching of basic asset pricing moments like the equity premium, 
the volatility of the equity premium, the variance premium, and excess return predictability by the price-dividend ratio and the variance risk premium. The important feature of our model from an economic standpoint is therefore that the probability of a large shock is not perfectly correlated to the 'usual' uncertainty represented by diffusive variance.

To provide a simple and very basic framework for why the dynamics of the IV smile in options markets allow us to study the different roles of diffusive and jump risk we refer to Yan (2011) who analyzes the link between level and slope in the context of a reduced-form model. He shows that for very short times to maturity the IV of an at-the-money option, i.e., the level of the smile, is proportional to the current value of the conditional volatility (i.e., the square root of diffusive variance), while the slope is approximately equal to the product of jump intensity and mean jump size, divided by the current conditional volatility. Making the intensity proportional to the conditional variance (squared volatility) would thus imply that the slope is a multiple of the conditional volatility and therefore locally perfectly correlated with the level. If this were true in the data we would expect the level and the slope of the IV smile to move almost in lockstep, and the slope should to be an affine function of the level. More important than the locally perfect correlation, however, is the fact that a specification with a jump intensity proportional to the conditional variance makes the slope of the smile a deterministic function of the level and vice versa (with an almost arbitrary functional form). We show below that this relation is strongly rejected by the data, while a model with a separation of jump intensity and variance generates patterns consistent with the stylized facts.

The arguments in Yan (2011) are strictly speaking only valid for infinitesimal time to maturity. To provide the intuition for longer maturities, we again resort to reduced-form option pricing models. Making the jump intensity an affine function of the conditional variance is a popular choice, see for example Bates (2000) and Pan (2002). It is clear that in such a model with the state variables $V$ 
(stochastic volatility) and $\lambda$ (stochastic intensity), the level $\nu$ and the slope $s$ of the IV smile will be uniquely determined given $V$ and $\lambda$. When $\lambda$ is an invertible function of $V$, i.e., when knowing $V$ is actually equivalent to knowing $\lambda$ (as it is the case in a model where $\lambda$ is affine in $V$ ), then level and slope have to move together in a one-to-one relation, i.e., knowing one implies knowing the other without error. The functional relationship between level and slope may be non-linear such that the usual linear correlation is only an imperfect measure for the degree of co-movement, but the key fact is that affine models with $V$ as the only independent state variable produce a tight link between level and slope.

To gain insight into the relation of the level and slope observed in the data consider Figure 1. It shows a scatter plot of the level and slope of the IV smile for 1-month options on the S\&P 500 index for the period from January 1996 to August 2015. The level is represented by the IV of an at-the-money option with moneyness (defined as strike price over index level) equal to 1, whereas the slope is given by the difference between the IVs of two options with moneyness values of 0.9 and 1 , respectively.

There are two important stylized facts in the context of our paper. First, the correlation between level and slope is negative (the estimate for our sample is -0.33$)^{1}$, and second, there is only a weak link between level and slope (a smoothing spline fit of level on slope yields an $R^{2}$ of only around 0.2$).^{2}$

Our model with separate processes for volatility and intensity is able to explain both of

\footnotetext{
${ }^{1}$ Alternative ways to measure the level and the slope of the implied volatility smile have been proposed in the literature. In Section E of the Online Appendix we provide a comparison of the results when we apply different measures for the two key quantities in our analysis. All our results are robust with respect to the actual approach taken to measure level and slope.

${ }^{2}$ Both of these facts are robust features of the data, as shown by a subperiod analysis in Section F of the online appendix. For the years from 1996 to 2007, e.g., the numbers are almost exactly the same as the ones shown above, with values of -0.34 for the correlation and 0.2 for $R^{2}$, respectively. For the period from 2009 to 2015 , the correlation is slightly more negative with a value of -0.46 , and the $R^{2}$ is a little higher (around 0.28), but the overall picture is very similar to what we obtain for the full sample. The online appendix contains further subperiod analyses, all of which indicate that the basic facts we have identified are robustly present in the data.
} 
these key properties of the data, while models in which the intensity is an affine function of the local variance are not. ${ }^{3}$ First, in simulated data our model generates confidence intervals for the correlation between level and slope that include negative values. In contrast to this, models in which the jump intensity is an affine function of variance produce consistently positive and large correlations. Second, our model reproduces the observed weak link between the level and slope, while the models with only one process driving both the variance and the intensity imply an almost perfect fit of a nonlinear regression of level on slope, which is strongly at odds with the data.

Both the negative correlation between level and slope and the fact that this correlation is low in absolute value are relevant, but the low explanatory power of slope for level (or vice versa) is the more important one. The reason for this is that different models and different parametrizations can potentially generate different signs for the correlation between level and slope. However, only our model with a separation of intensity from variance can reproduce the low explanatory power of the slope for the level observed in the data.

Furthermore, the weak link between level and slope is not just of technical interest in the context of the asset pricing model discussed in this paper, but, as mentioned above, the two risk types related to level and slope are fundamentally economically different. When we look at their respective correlations with macro variables, we find quite different patterns for level and slope. McCracken and $\mathrm{Ng}$ (2015) provide a cross-section of 134 monthly macro time-series categorized into eight groups like 'Output and Income', 'Labor Market', 'Consumption and Orders' etc. Table 1 provides the results of the regressions of level and slope on the respective (standardized) first principal components for the eight groups of variables. Furthermore, we include two popular variables measuring economy-wide uncertainty, namely the uncertainty factor JLN from Jurado, Ludvigson, and Ng (2015) (for $h=1$

\footnotetext{
${ }^{3}$ We are not claiming that the separation of intensity and variance is the only way to break the relation between level and slope. In the class of long-run risk models, however, it is a straightforward solution.
} 
in their notation) and the liquidity factor PS suggested by Pástor and Stambaugh (2003).

Overall we find that both level and slope are related to macro variables, and it is interesting to note that more than 60 percent of the variation in level can be explained by a linear combination of the right-hand side variables. For slope, the share of explained variation is substantially lower, but this is also what one would expect, given that the overall level of stock market volatility is often linked to general economic uncertainty (see, e.g., Bloom (2009)), while slope with its closer ties to jump risk is certainly less 'linear' than level. It is in line with this argument that level is significantly related to the two general uncertainty variables JLN and PS, while for slope we do not see a significant relationship here.

Also in terms of a significant impact of the eight groups of variables there are clear differences between level and slope. For those variables which have a significant impact on both level and slope, the signs of the coefficients are different. For example, level is significantly positively related to the first principal component of macroeconomic measures for 'Output and income', while slope exhibits a significantly negative coefficient here. For 'Consumption and housing' and 'Interest rates and exchange rates' we observe the opposite, with negative coefficients for level and positive ones for slope. Furthermore, there are also cases, when only one of the two option measures loads significantly on the respective macro groups of variables. For example, in the regression for level the coefficient for 'Prices' is significant, while it is not statistically different from zero for slope, and we find the same for the first principal component related to the stock market.

We take these findings as strong evidence that level and slope are indeed two different phenomena. We thus separate the jump intensity from the conditional variance and rely on two different processes in the long-run risk model to model variance and jump intensity.

Following the previous literature we also include a stochastic mean reversion level of volatility 
and intensity as a state variable in our model. The presence of this variable, however, does not change the picture very much. Consider the upper left and upper right graph in Figure 2, which show modelgenerated values for level and slopes as well as non-parametrically estimated regression lines for our model (including such a stochastic mean-reversion level) and an otherwise identical model where intensity is affine in variance. It is immediately obvious that the model with separate processes (left graph) is able to reproduce the weak link between level and slope, while the other one (right graph) is not, since there the slope is almost a deterministic function of the level. As argued above, without the stochastic mean-reversion level for variance and thus also intensity, this link would even be perfect.

A comparison of Figures 1 and 2 shows that all model specifications generate an implied volatility slope that is slightly lower than observed in the data. This is due to the fact that the equilibrium specifications analyzed in this paper abstract from market microstructure factors that are relevant for the explanation of implied volatility smiles. For example, Bollen and Whaley (2004) show that the excess demand especially for out-of-the-money put options can induce a significant upward move in their implied volatilities. Similarly, in their theoretical model Gârleanu, Pedersen, and Poteshman (2009) show that the demand for options is an important determinant of their prices and IVs, with the effect being especially pronounced for OTM options.

The assumption of perfect correlation between intensity and variance is also called into question in other papers. For example, using reduced-form models, Santa-Clara and Yan (2010) and Christoffersen, Jacobs, and Ornthanalai (2012) show that volatility and jump intensity are not linked very tightly. Christoffersen, Feunou, Jeon, and Ornthanalai (2016) find that the market crash probability is only weakly related to return variance after controlling for the market illiquidity factor. This implies that it is illiquidity, not volatility, that determines the intensity of jumps. Further, Shaliastovich (2015) finds that macro confidence jump risks can play a crucial role in explaining the 
variance risk premium and volatility surface. Finally, Cremers, Halling, and Weinbaum (2015) show that aggregate jump and volatility risks are priced differently in the cross-section of stock returns.

Given that a key topic of our paper is to identify the separate roles of jump and volatility risk, it is natural to ask how they contribute in relative terms to the risk premia in the model. With respect to this criterion, jump risk appears to be more important than diffusive risk, which is probably not overly surprising, given the large literature on disaster risk as a main driver of the equity risk premium. In our benchmark calibration the total equity risk premium amounts to slightly more than $6 \%$, with about two thirds coming from jump and one third coming from diffusive risk. Within the two groups, diffusive risk is mostly relevant for the expected growth rate of consumption, whereas with respect to jumps it is mostly the fact that the conditional variance exhibits jumps, which drives this part of the premium.

It may even be more intuitive to look at the representation of the diffusive and jump-related parts of the equity premium in terms of the current values of the different state variables. For example, in our benchmark specification the diffusive part of the equity premium loads most strongly on the current value of the long-term mean of variance, while the jump part is entirely given as a multiple of the current value of the intensity process.

A further, albeit slightly more technical, contribution of our paper is that we propose a square root (SQR) process for the stochastic mean reversion level of the conditional variance and the jump intensity, in contrast to Drechsler and Yaron (2011), who use Ornstein-Uhlenbeck (OU) dynamics. The main reason for this choice is that the SQR process theoretically remains positive, which is a desirable feature for a long-term variance level.

We do not want to over-emphasize the general relevance of this model variation, but it improves the performance of the model to a certain degree in various dimensions. For one, it brings 
about a lower $R^{2}$ in the regression of levels on slopes, which becomes evident from a comparison of the SQR and the OU specifications in Figure 2. Second, it somewhat improves the performance the model in predictability regressions. Finally, it leads to an equity premium which is the higher, the higher the conditional long-term mean of volatility, while in the OU case, the long-term mean of volatility does not play a role here.

Our paper is related to several strands of the literature. First of all, our approach is in the tradition of LRR models pioneered by Bansal and Yaron (2004), who successfully apply them to the pricing of the aggregate stock market and to the analysis of predictability relationships between variables like the current price-dividend ratio and future excess returns on equity. Since then models of this type have been applied in a variety of asset pricing contexts. Examples are the papers by Bansal, Gallant, and Tauchen (2007), Kiku (2006), Malloy, Moskowitz, and Vissing-Jorgensen (2009), Bansal and Shaliastovich (2011), Kaltenbrunner and Lochstoer (2010) and Colacito and Croce (2011), to name just a few. Bansal, Dittmar, and Lundblad (2005) and Hansen, Heaton, and Li (2008) apply LRR models in order to explain cross-sectional variation in equity returns, while Hasseltoft (2012) and Bansal and Shaliastovich (2013) use the LRR framework to explain return dynamics in bond markets. $^{4}$

Most relevant to our research question are two papers which apply the LRR framework to derivative securities. Benzoni, Collin-Dufresne, and Goldstein (2011) extend the basic LRR framework by introducing jumps in the state variables and use their model to explain the emergence of the implied volatility skew for options on the S\&P 500 index after the crash of 1987. Drechsler and Yaron (2011) rely on a time-varying mean reversion level of the stochastic volatility component and also include jumps with random intensities, but assume that these are affine in the conditional

\footnotetext{
${ }^{4}$ Besides the theoretical papers which consider the ability of LRR models to explain stylized facts in the data there is also a growing literature concerned with the empirical performance of the model, e.g., Beeler and Campbell (2012), Constantinides and Ghosh (2011), Bansal, Kiku, and Yaron (2012), and Jagannathan and Marakani (2011).
} 
variance.

\section{Model Setup}

\subsection{The Investor}

We assume a continuous-time endowment economy with a single perishable consumption good. The representative agent has recursive preferences of the Epstein and Zin (1989) type with stochastic differential utility $J$ given by

$$
J_{t}=E_{t}\left[\int_{t}^{\infty} f\left(C_{s}, J_{s}\right) d s\right]
$$

Here $C_{t}$ is the investor's consumption at time $t$, and $f$ denotes the aggregator function

$$
f(C, J)=\frac{\beta C^{1-\frac{1}{\psi}}}{\left(1-\frac{1}{\psi}\right)[(1-\gamma) J]^{\frac{1}{\theta}-1}}-\beta \theta J
$$

$\beta$ is the investor's subjective time preference rate. The parameter $\theta$ is defined as $\frac{1-\gamma}{1-\frac{1}{\psi}}$, where $\psi \neq 1$ and $\gamma \neq 1$ denote the intertemporal elasticity of substitution (IES) and the relative risk aversion, respectively. In the following we will assume $\psi>1$ and $\gamma>1$.

The recursive utility specification allows the level of relative risk aversion and the IES to be chosen independently of each other. For the special case $\gamma=\frac{1}{\psi}$, the preferences in equations (1) and (2) collapse to the usual power utility specification where the IES is just the inverse of relative risk

aversion. In the case where $\gamma>\frac{1}{\psi}$ the investor is said to have a preference for early resolution of uncertainty. 


\subsection{The Economy}

Our economy is described by the processes for consumption and dividends, as well as for the state variables governing the future cash flow dynamics. The dynamics of consumption $C$ and dividends $D$ are given by

$$
\begin{aligned}
& d \ln C_{t}=\left(\mu_{c}+x_{t}\right) d t+\sqrt{g_{c c, t}} d W_{t}^{c} \\
& d \ln D_{t}=\left(\mu_{\delta}+\phi x_{t}\right) d t+\sqrt{g_{\delta \delta, t}}\left(\rho_{t} d W_{t}^{c}+\sqrt{1-\rho_{t}^{2}} d W_{t}^{\delta}\right) .
\end{aligned}
$$

$W^{c}$ and $W^{\delta}$ denote two independent Wiener processes. The long-run risk factor $x$ captures the deviation of the conditionally expected consumption growth from its long-run mean $\mu_{c}$. As usual in the LRR literature (see, e.g., Bansal and Yaron (2004)) $\phi>1$ plays the role of a 'leverage factor', since dividends, which are paid by (presumably levered) firms, have a larger exposure to changes in the economic environment than consumption.

The variances $g_{c c}$ and $g_{\delta \delta}$ of innovations in consumption and dividends are stochastic, and we set

$$
g_{j j, t}=\sigma_{j}^{2}\left(w_{j} \sigma_{t}^{2}+1-w_{j}\right) \quad j \in\{c, \delta\} .
$$

The weights $w_{j} \in[0,1]$ for $j \in\{c, \delta\}$ determine to what degree the conditional variance of the respective variable is affected by the conditional variance process $\sigma^{2}$, which has a long-run mean equal to one. In our parametrization we set $w_{c}=0.5$ and $w_{\delta}=0.125$. The conditional correlation $\rho_{t}$ between consumption and dividend innovations follows from the local conditional covariance $g_{c \delta}$ 
given as

$$
g_{c \delta, t}=w_{c \delta} \sigma_{c} \sigma_{\delta}\left(\sqrt{w_{c} w_{\delta}} \sigma_{t}^{2}+\sqrt{\left(1-w_{c}\right)\left(1-w_{\delta}\right)}\right) .
$$

The dynamics of consumption and dividends depend on the classical long-run growth factor $x$ and on the stochastic variance of consumption growth $\sigma^{2}$. In terms of state variables there are also the 'intensity factor' $\alpha$, and the time-varying mean reversion level for the processes $\sigma^{2}$ and $\alpha$, denoted by $\bar{\sigma}^{2}$. As mentioned above, the introduction of $\alpha$ as a separate driver for the stochastic jump intensity is the key innovation in our model with respect to approaches like Drechsler and Yaron (2011). ${ }^{5}$ The dynamics of the state variables are

$$
\begin{aligned}
d x_{t} & =-\kappa_{x} x_{t} d t+\sqrt{g_{x x, t}} d W_{t}^{x}+\xi^{x} d N_{t}^{x}-E\left[\xi^{x}\right] l_{x, t} d t \\
d \sigma_{t}^{2} & =k_{\sigma, \bar{\sigma}}\left(\bar{\sigma}_{t}^{2}-\sigma_{t}^{2}\right) d t+\sqrt{g_{\sigma \sigma, t}} d W_{t}^{\sigma}+\xi^{\sigma} d N_{t}^{\sigma}-E\left[\xi^{\sigma}\right] l_{\sigma, t} d t \\
d \alpha_{t} & =k_{\alpha, \bar{\sigma}}\left(\bar{\sigma}_{t}^{2}-\alpha_{t}\right) d t+\sqrt{g_{\alpha \alpha, t}} d W_{t}^{\alpha}+\xi^{\alpha} d N_{t}^{\alpha}-E\left[\xi^{\alpha}\right] l_{\alpha, t} d t \\
d \bar{\sigma}_{t}^{2} & =\kappa_{\bar{\sigma}}\left(\overline{\bar{\sigma}}^{2}-\bar{\sigma}_{t}^{2}\right) d t+\sqrt{g_{\bar{\sigma} \bar{\sigma}, t}} d W_{t}^{\bar{\sigma}},
\end{aligned}
$$

where $W^{x}, W^{\sigma}, W^{\alpha}$, and $W^{\bar{\sigma}}$ are independent Wiener processes. $N^{x}, N^{\sigma}$, and $N^{\alpha}$ represent three independent jump processes whose intensities depend on $\sigma_{t}^{2}$ and on the intensity factor $\alpha$. We set the drift terms such that the long run mean of $x_{t}$ is zero, while the long run means of the three uncertainty variables are equal to one. ${ }^{6}$ Analogous to $g_{c c, t}$ and $g_{\delta \delta, t}$, the conditional variances of the

\footnotetext{
${ }^{5}$ The role of $\alpha$ in our model will be discussed in detail below.

${ }^{6}$ Details are given in Online Appendices A and B.
} 
state variables are given as

$$
\begin{aligned}
& g_{x x, t}=\sigma_{x}^{2}\left(w_{x} \sigma_{t}^{2}+1-w_{x}\right) \\
& g_{\sigma \sigma, t}=\sigma_{\sigma}^{2}\left(w_{\sigma} \sigma_{t}^{2}+1-w_{\sigma}\right) \\
& g_{\alpha \alpha, t}=\sigma_{\alpha}^{2}\left(w_{\alpha} \alpha_{t}+1-w_{\alpha}\right) \\
& g_{\bar{\sigma} \bar{\sigma}, t}=\sigma_{\bar{\sigma}}^{2}\left(w_{\bar{\sigma}} \bar{\sigma}_{t}^{2}+1-w_{\bar{\sigma}}\right) .
\end{aligned}
$$

Setting $w_{\sigma}=w_{\alpha}=1$, as we will do below, implies that both $\sigma^{2}$ and $\alpha$ follow classical square-root processes (albeit with jumps).

The choice of $w_{\bar{\sigma}}$ is of major importance and determines a key feature of the model, namely if $\bar{\sigma}$ follows an OU process $\left(w_{\bar{\sigma}}=0\right)$, or if this variable is governed by an SQR process $\left(w_{\bar{\sigma}}=1\right) .^{7}$ It is obvious from both an economic and a mathematical point of view that a process describing the mean-reversion level of the non-negative processes $\sigma^{2}$ and $\alpha$ should be non-negative itself. This is not the case for an OU process, so that this specification violates fundamental modeling restrictions. This technical aspect is, however, not the only reason why the choice for $w_{\bar{\sigma}}$ matters. It is also relevant for the properties of the expected excess return on the consumption and the dividend claim (see Section 3), and for the predictability generated by the model (see Section 5.2.2).

We will now discuss the jump part of the model in more detail. The long-run growth factor $x$, the stochastic variance $\sigma^{2}$, and the intensity factor $\alpha$ are subject to jumps. As usual we assume that there are no simultaneous jumps. Note that consumption or dividends do not themselves jump. Our approach thus differs from the one presented in the literature on disaster risk, pioneered by Rietz

\footnotetext{
${ }^{7}$ Egloff, Leippold, and Wu (2010) also employ a square-root process for the mean reversion level of the conditional variance. However, their model is focused on the properties of volatility as an asset class and not on the equilibrium characteristics of this variable. Examples for two-factor volatility models are presented in Zhou and Zhu (2012) and Bates (2000), among others. In these models, it is not the mean reversion level, which is modeled as the second factor, but the conditional variance is the sum of two processes, usually both of the square-root type.
} 
(1988) and further developed by, among others, Barro (2009) and Backus, Chernov, and Martin (2011). There the cash flow variable itself can be subject to large and sudden downward shocks, so that the consumption claim becomes a very risky asset commanding a high risk premium. ${ }^{8}$

We assume that jumps in $x$ are normally distributed with mean $\mu_{\xi^{x}}$ and volatility $\sigma_{\xi^{x}}$. Jumps in $\sigma^{2}$ and $\alpha$ have to be positive, so that we assume exponential distributions with means $\mu_{\xi^{\sigma}}$ and $\mu_{\xi^{\alpha}}$, respectively. The intensities $l_{x, t}, l_{\sigma, t}$ and $l_{\alpha, t}$ of the jumps are assumed to be affine functions of the state variables $\sigma_{t}^{2}$ and $\alpha_{t}$ :

$$
l_{i, t}=l_{i 0}+l_{i 1, \sigma} \sigma_{t}^{2}+l_{i 1, \alpha} \alpha_{t} \quad i \in\{x, \sigma, \alpha\}
$$

We are especially interested in the roles of $\sigma^{2}$ and $\alpha$ as factors driving jump intensities. As a simple way to shed light on this we further assume that the coefficients $l_{i 1, \sigma}$ and $l_{i 1, \alpha}$ are both multiples of some constant $\hat{l}_{i 1}$ with $l_{i 1, \sigma}=\left(1-\varphi_{\alpha}\right) \hat{l}_{i 1}$ and $l_{i 1, \alpha}=\varphi_{\alpha} \hat{l}_{i 1}$, so that the final specification for the intensity processes is

$$
l_{i, t}=l_{i 0}+\left(1-\varphi_{\alpha}\right) \hat{l}_{i 1} \sigma_{t}^{2}+\varphi_{\alpha} \hat{l}_{i 1} \alpha_{t} \quad i \in\{x, \sigma, \alpha\} \text {. }
$$

Motivated by stylized facts from the market for index options, the introduction of the factor $\alpha$ is the key innovation in our setup compared to the existing literature. Restricted versions of the general model are then obtained by simply setting $\varphi_{\alpha}$ to special values. With $\varphi_{\alpha}=1$ the jump intensity is locally independent of $\sigma^{2}$ and driven only by the new intensity factor $\alpha$. On the other hand, setting $\varphi_{\alpha}=0$ yields a model, where the jump intensity is only driven by the conditional variance $\sigma^{2}$, which corresponds to the popular specification in reduced-form option pricing models

\footnotetext{
${ }^{8}$ See also Juillard and Ghosh (2012) for a critical analysis of the role of consumption crashes as the source of the equity premium.
} 
like Bates (2000) or Pan (2002) and to the setup in Drechsler and Yaron $(2011)^{9}$. $0<\varphi_{\alpha}<1$ represents an intermediate case. ${ }^{10}$

From a theoretical modeling perspective, having a second potential driver for jump intensities in addition to the conditional variance makes it possible to actually distinguish between jump and diffusive risk. With $\varphi_{\alpha}=0$ an increase in the conditional variance automatically implies an increase in the risk of a jump occurring over the next time interval, so that a 'normal' increase in uncertainty (higher conditional diffusive variance) would always automatically imply a higher risk of dramatic changes in the state variables (higher jump intensity). With $\varphi_{\alpha} \in(0 ; 1]$ on the other hand there may be an increase in jump risk without a simultaneous increase in diffusion risk, and vice versa.

\section{The Equilibrium}

\subsection{Equity Risk Premium}

In this section we summarize the equilibrium solution for our model. ${ }^{11}$ We will first derive the expression for the equity risk premium.

The dynamics of the pricing kernel in continuous time are given by

$$
d \ln M_{t}=-\theta \beta d t-\frac{\theta}{\psi} d \ln C_{t}-(1-\theta) d \ln R_{c, t}
$$

where $d \ln R_{c, t}$ is the log-return on the consumption claim at time $t$. To solve for this log-return, Eraker and Shaliastovich (2008) approximate it via log-linearization following Campbell and Shiller

\footnotetext{
${ }^{9}$ Benzoni, Collin-Dufresne, and Goldstein (2011) also consider stochastic jump intensities, but in their model the jump intensity follows a purely exogenous two-state Markov chain.

${ }^{10}$ In our numerical exercise we do not calibrate $\varphi_{\alpha}$, but only consider the special cases $\varphi_{\alpha} \in\{0,1\}$.

${ }^{11} \mathrm{~A}$ detailed derivation of the equilibrium in an affine jump-diffusion model can be found in Eraker and Shaliastovich (2008).
} 
(1988):

$$
d \ln R_{c, t}=k_{0} d t+k_{1} d v_{t}-\left(1-k_{1}\right) v_{t} d t+d \ln C_{t} .
$$

$v_{t}$ is the $\log$ wealth-consumption ratio, and $k_{0}$ and $k_{1} \in(0,1)$ are called 'linearization constants', which depend on the average log wealth-consumption ratio (see Online Appendix C.1 for details). $v_{t}$ is assumed to be affine in $Y_{t}=\left(x_{t}, \sigma_{t}^{2}, \alpha_{t}, \bar{\sigma}_{t}^{2}\right)^{\prime}$, i.e.

$$
v_{t}=A_{0}+A_{1}^{\prime} Y_{t}
$$

where $A_{0}$ and $A_{1}$ together with $k_{0}$ and $k_{1}$ solve a non-linear system of equations (see again Online Appendix C.1). With Epstein-Zin preferences and $\psi>1$ one obtains $A_{1 x}>0$, where $A_{1 x}$ is the component related to $x$ in the vector $A_{1}$. With $\gamma$ and $\psi$ both greater than $1, A_{1 \sigma}, A_{1 \alpha}$, and $A_{1 \bar{\sigma}}$ are all negative (see again Online Appendix C.1).

Once we know the pricing kernel $M,^{12}$ we can price any other claim, given its future payoffs. The log return on the dividend claim can be approximated like the log return on the consumption claim:

$$
d \ln R_{\delta, t}=k_{\delta 0} d t+k_{\delta 1} d v_{\delta, t}-\left(1-k_{\delta 1}\right) v_{\delta, t} d t+d \ln D_{t}
$$

where $v_{\delta, t}$ denotes the $\log$ price-dividend ratio ( $\mathrm{p}$-d ratio hereafter), again assumed to be affine in

\footnotetext{
${ }^{12}$ See Online Appendix C.3.
} 
$Y_{t}::^{13}$

$$
v_{\delta, t}=A_{\delta 0}+A_{\delta 1}^{\prime} Y_{t}
$$

The expected excess return on the dividend claim (i.e, the equity premium) follows from the exposures to the risk factors and the market prices of risk. It is given as

$$
\begin{aligned}
\frac{E\left[d \ln R_{\delta, t}\right]}{d t}-r_{t}= & \gamma g_{c \delta, t}+\sum_{i=x, \sigma^{2}, \alpha, \bar{\sigma}^{2}}(1-\theta) k_{\delta 1} A_{\delta 1 i} g_{i i, t} k_{1} A_{1 i}-\frac{1}{2} \frac{\operatorname{Var}\left[d \ln R_{\delta, t}^{\text {cont }}\right]}{d t} \\
& +\sum_{i=x, \sigma, \alpha} l_{i t} C_{i},
\end{aligned}
$$

where $d \ln R_{\delta, t}^{\text {cont }}$ denotes the continuous part of the return, and

$$
C_{i}=k_{\delta 1} A_{\delta 1 i} E\left[\xi^{i}\right]+E\left[\exp \left(-(1-\theta) k_{1} A_{1 i} \xi^{i}\right)\right]-E\left[\exp \left(\left(k_{\delta 1} A_{\delta 1 i}-(1-\theta) k_{1} A_{1 i}\right) \xi^{i}\right)\right]
$$

for $i \in\{x, \sigma, \alpha\} . A_{1 i}$ and $A_{\delta 1 i}$ are the components of the vectors $A_{1}$, and $A_{\delta 1}$ associated with variable $i$.

The first two terms on the right-hand side of equation (12) give the premia for diffusive risk, while the third term represents a Jensen correction term. The three terms of the sum in the second line are the premia for jump risk in $x, \sigma$, and $\alpha$. In more detail, the first term on ther right-hand side of equation (12), $\gamma g_{c \delta, t}$, is the premium for consumption diffusion risk. As with CRRA it is equal to the product of relative risk aversion $\gamma$ and the covariance of dividend risk with consumption risk, $g_{c \delta, t}$ (see equation (4)). With Epstein-Zin preferences, state variables are also priced. This part of the equity risk premium depends on the exposure of the dividend claim and of the consumption claim

\footnotetext{
${ }^{13}$ Details on the computation of the coefficients $A_{\delta 0}$ and $A_{\delta 1}$ and the linearization constants $k_{\delta 0}$ and $k_{\delta 1}$ are given in Online Appendix C.2.
} 
to these variables, where the latter determine the market prices of risk.

Given $\gamma>\frac{1}{\psi}$, the sensitivities of the $\mathrm{p}$-d ratio and the wealth-consumption ratio to the state variables are in line with intuition. Both valuation ratios increase with $x$ and decrease with the uncertainty factors. The premium on the long-run growth rate $x$ is then positive, since both the exposure of the stock price and the market price of risk are positive. The premia on the uncertainty factors $\sigma^{2}, \alpha$, and $\bar{\sigma}^{2}$ are also positive, since the negative exposures are multiplied by negative market prices of risk. Put together, the premia on all diffusive risk factors are positive.

The diffusion risk premia are proportional to the local diffusion (co)variances $g_{c \delta, t}$ and $g_{i i, t}$. Since the covariance $g_{c \delta, t}$ and the variances $g_{x x, t}$ and $g_{\sigma \sigma, t}$ are increasing in $\sigma^{2}$, the expected excess return also increases with $\sigma^{2}$. When the jump intensities and the conditional variance are not perfectly correlated, $\alpha$ also has an impact on asset prices, so that with the variance $g_{\alpha \alpha, t}$ increasing in $\alpha$ the equity premium increases in $\alpha$ as well. With our SQR specification the equity risk premium increases with $\bar{\sigma}^{2}$, since the conditional variance $g_{\bar{\sigma} \bar{\sigma}}$ increases in $\bar{\sigma}_{t}^{2}$. For the OU specification considered in Drechsler and Yaron (2011) the expected excess return on the divindend claim is independent of the mean reversion level, since here $g_{\bar{\sigma} \bar{\sigma}}$ is constant.

The premia for jumps in $x, \sigma$ and $\alpha$ depend on the exposures $k_{\delta 1} A_{\delta 1}$ of the p-d ratio to the state variables, on the market prices of risk, and on the jump intensities. Jumps in $x$ are more frequent and more negative under the risk-neutral than under the true measure. ${ }^{14}$ Together with the fact that the price of the dividend claim is increasing in $x$, this results in a positive contribution of the jump-related premium for $x$ to the total risk premium. A similar argument shows that the premia for jumps in $\sigma^{2}$ and $\alpha$ have to be positive, since the $\mathrm{p}$-d ratio loads negatively on these two variables, their jump intensities are higher under $\mathbb{Q}$ than under $\mathbb{P}$, and the mean jump size is greater under the risk-neutral than under the physical measure.

\footnotetext{
${ }^{14}$ See Online Appendix C.4.
} 


\subsection{Variance Risk Premium and Option Pricing}

The variance risk premium $V R P_{t}$ at time $t$ is defined as the difference of the expected quadratic variation of the return over a time interval $\tau$ under the risk-neutral measure $\mathbb{Q}$ and the physical measure $\mathbb{P}$, i.e.,

$$
V R P_{t}=E_{t}^{\mathbb{Q}}\left[\int_{t}^{t+\tau}\left(d \ln R_{\delta, s}\right)^{2}\right]-E_{t}^{\mathbb{P}}\left[\int_{t}^{t+\tau}\left(d \ln R_{\delta, s}\right)^{2}\right]
$$

Details on its calculation are given in Online Appendix C.5.

A positive variance risk premium implies that the investor is actually willing to accept an expected return below the risk-free rate on an asset with positive exposure to variance risk. ${ }^{15}$

As described in the introduction the dynamics of the IV smile are the key object which helps us to separate changes in jump risk from changes in diffusion risk. It is therefore necessary to compute option prices in our model. The above analysis has equipped us with the $\mathbb{Q}$-dynamics of all cash flow quantities and state variables, from which we can then deduce the $\mathbb{Q}$-dynamics of the stock price.

The price $C_{t}$ of a European call with maturity date $t+\tau$ and strike price $k$ is then given as

$$
C_{t}=E_{t}^{\mathbb{Q}}\left[e^{-\int_{t}^{t+\tau}\left(r_{0}+r_{1}^{\prime} Y_{s}\right) d s}\left(S_{t+\tau}-k\right)^{+}\right]
$$

where $S_{t}$ is the price of the dividend claim at time $t$ given by $S_{t}=D_{t} e^{v_{\delta, t}}$. The computation of a European option price then only requires the extra step of computing a Fourier inversion along the lines of Duffie, Pan, and Singleton (2002). ${ }^{16}$

\footnotetext{
${ }^{15}$ Examples for papers dealing with the variance risk premium are Bollerslev, Sizova, and Tauchen (2012), Bollerslev, Tauchen, and Zhou (2010), Carr and Wu (2009), Egloff, Leippold, and Wu (2010), and Todorov (2010).

${ }^{16}$ Details are given in Online Appendix C.6.
} 


\section{Data}

The empirical facts we want to match and explain with our model relate to a number of key asset pricing moments like the equity risk premium, represented by the average excess return on the S\&P 500 index, the risk free rate, represented by the return on three month T-bills, the variance risk premium, and the level and the slope of the IV smile for options on the S\&P 500 index. Furthermore, we also try to match dynamic properties of the data, such as the predictability of future excess returns, consumption and dividend growth, as well as the co-movement of the level and the slope of the IV smile.

The data come from a variety of sources. Consumption data are taken from the National Income and Product Accounts (NIPA) published by the Bureau of Economic Activity (BEA). As our consumption measure we use real personal per capita consumption of non-durables and services (NIPA Table 7.1). As stated above we take the S\&P 500 index as the representative for the dividend claim in our model. Data on the S\&P 500 index are taken from the Center of Research in Security Prices (CRSP). The time series for dividends is constructed using the difference in index returns with and without dividends as proposed Bansal, Dittmar, and Lundblad (2005) and applied in, e.g., Beeler and Campbell (2012). The return data and the dividend series are converted to real figures using the consumer price index (CPI). The risk-free rate is constructed using data on three month T-bills provided by the Federal Reserve. ${ }^{17}$ Since the ex-ante real risk free rate is not observable we follow the procedure used, e.g., in Beeler and Campbell (2012) and approximate it by the fitted values from a regression of the ex-post real rate on the current nominal rate and inflation. Inflation is again measured as the growth rate of the CPI. We use yearly data from 1930 until 2015.

For the computation of the variance risk premium in month $t$ we use daily returns on the $\mathrm{S} \& \mathrm{P}$

\footnotetext{
${ }^{17}$ We convert the T-bill data from a discount base to a return base.
} 
500 and daily values for the VIX index taken from the Chicago Board Options Exchange (CBOE). The expected integrated variance under the $\mathbb{Q}$-measure for month $t$ is approximated by the squared VIX at the end of month $t-1$. Under the $\mathbb{P}$-measure the expected integrated variance for month $t$ is taken to be the forecast from an $\operatorname{ARMA}(1,1)$ model for the realized monthly integrated variance, which is computed as the sum of the squared daily returns on the S\&P 500 within each month. The sample period for the variance risk premium ranges from January 1990 until December 2015.

Options data are taken from OptionMetrics for the period from January 1996 to August 2015. We use end-of-the-month prices for put options with a trading volume of at least 100 contracts on the last day of the respective month. To obtain an estimate of the implied volatilities for our representative options with a time to maturity of 30 days and moneyness levels (defined by strike divided by spot price) of 0.9 and of 1.0, respectively, we use a two dimensional linear interpolation scheme. ${ }^{18}$

The model numbers corresponding to these empirical data are generated via Monte Carlo simulation. To simulate the dynamics of the vector $Y$ we use a first-order Euler discretization for the system of stochastic differential equations with a discretization step of one day. Jumps are simulated by drawing from the Poisson distribution with the current value of the stochastic intensity. The daily observations generated by our simulation procedure are then aggregated to the same frequency as the corresponding data. This means we aggregate consumption growth, dividend growth, the return on the dividend claim, and the risk free rate to yearly data, and the information on the variance risk premium and the option IVs to monthly data. We compute the simulation output for the model such that the time span matches the length of observations in the data, i.e., 86 years for the yearly

\footnotetext{
${ }^{18}$ There are of course different ways to compute the slope of the implied volatility curve besides linearly interpolating between two points on the volatility curve. One alternative is the the regression-based measure suggested by Foresi and $\mathrm{Wu}$ (2005). In Onlie Appendix E we show that our results are robust with respect to different ways of measuring the slope of the IV smile.
} 
information, 312 months for the variance risk premium, and 236 months for the option prices.

To determine the dynamic relationship between the level and the slope of the IV smile we use two measures. The first is the standard (linear) correlation between the two variables, and the second is the $R^{2}$ from fitting a nonlinear spline to slope as a function of level. Since specifications without the separate intensity process tend to generate tight fits of slope as a function of levels, we choose the smoothing parameter in the spline to favor high $R^{2} \mathrm{~s}$, so that it is harder for our model to differentiate itself from approaches where intensity is affine in variance. ${ }^{19}$

\section{$5 \quad$ Numerical Results}

\subsection{Calibration}

In the following analysis we compare four versions of our model, which are distinguished by the set of state variables included (with or without a separate intensity factor $\alpha$ ) and by the dynamics of the stochastic mean reversion level for the conditional variance (following an OU or an SQR process).

What we call the 'full' model is the one including $\alpha\left(\varphi_{\alpha}\right.$ is set to 1 in equation (7)) and where $\bar{\sigma}^{2}$ follows a square-root process (indicated by $w_{\bar{\sigma}}=1$ in equation (6)). In the other versions either one or both of these features are not present. The model 'Without $\alpha$, SQR' is one where $\alpha$ is not present $\left(\varphi_{\alpha}=0\right)$ and $\bar{\sigma}^{2}$ follows an SQR process $\left(w_{\bar{\sigma}}=1\right)$. The labels 'With $\alpha$, OU' and 'Without $\alpha$, OU' are to be understood accordingly. The specification 'Without $\alpha$, OU' denotes the model analyzed in Drechsler and Yaron (2011), and we will use this specification as our benchmark.

We follow the methodology given in Drechsler and Yaron (2011) for the parametrization of the models and calibrate them to match a broad set of moments as closely as possible. ${ }^{20}$

\footnotetext{
${ }^{19}$ Details on the smoothing spline method can be found in Online Appendix D.

${ }^{20}$ There are some papers which actually estimate LRR models, e.g., Bansal, Kiku, and Yaron (2012), Constantinides and Ghosh (2011), Hasseltoft (2012), or Zhou and Zhu (2015). However, the dynamics of the cash flow and state
} 
The annualized parameters for all four model versions are shown in Table 2. ${ }^{21}$ Our benchmark calibration is the case denoted by "Without $\alpha$, OU", which is the continuous time version of the model analyzed in Drechsler and Yaron (2011), and for this specification we take their parameters. ${ }^{22}$ The same holds true for the case "Without $\alpha$, SQR".

For the specifications with $\alpha$ we first set the paramters for $\alpha$ equal to the parameters for $\sigma^{2}$.

Since the separation of intensity and conditional variance, which is the key feature of our model, results in an overall reduction of risk in the economy due to a diversification effect, we need to recalibrate the model compared to Drechsler and Yaron (2011). We perform this recalibration step by step, changing the dynamics of one state variable at a time. After each step, we report the calibration results for a subset of asset pricing moments. The parameters used in each step can be found in Table 3, the asset pricing moments resulting from the parametrization are given in Table 4.

First, we introduce the intensity process $\alpha$ and set its parameters equal to those for $\sigma^{2}$ (Panel A). The processes for the intensity and the stochastic diffusion variance are thus the same as in the benchmark model of DY, with the only exception that they are no longer perfectly correlated. Second we change the parameters for $\sigma$ and also lower risk aversion from 10 to 8.8 (Panel B). Third, we further adjust the the mean reversion speed $\kappa_{\bar{\sigma}}$ of the long-run mean-reversion level $\bar{\sigma}^{2}$ (Panel C). Fourth, we change the parameters of the stochastic intensity process $\alpha$, which finally results in the calibration of the full model (Panel D).

We restrict the analysis of this exercise to a subset of cash flow and asset pricing moments considered later in Section 5.2 below. In Table 4, we show results for dividend and consumption variables in these models are rather restricted compared to ours. The moments we want to match are the dynamics of consumption and dividends, the equity risk premium, and the predictability of stock returns by the price-dividend ratio. Furthermore, we aim at the variance risk premium, the predictability of stock returns by the variance risk premium, and, last but not least, the dynamics of level and slope of the volatility smile.

${ }^{21}$ For the technical details on the calibration of the model see Online Appendix G.

${ }^{22}$ Note that Drechsler and Yaron (2011) show the parameters in monthly frequency. Further, we use a normal distribution for the jump size of $x$, so that the calibration follows the information given in Table 5 and Table 11 of their paper. 
growth, the equity risk premium, the variance risk premium, option moments for contracts with one month to maturity, and predictability results.

The introduction of the separate process $\alpha$ for the stochastic intensity reduces the overall risk in the economy when we keep the parametrization as in the benchmark specification. This can be seen by inspecting the results in the columns of the panel "DY Calibration" of Table 4 . There is a slight decrease in the standard deviation of dividends and consumption compared to the data. This diversification effect is also clearly noticeable in the results for the asset pricing moments. The values for the equity premium and the variance risk premium are too low, as are their standard deviations. It is interesting to notice that the variance risk premium is all but nonexistent in this calibration. Concerning the option prices both the level and the slope of the IV smile are too low. The only immediate improvement as compared to the benchmark model is the relation between the level and slope of the smile. The correlation between these two moments goes down and is now centered at about zero. More importantly, the $R^{2}$ for the spline fit between level and slope is much lower now. In fact, the $95 \%$-quantile is now even lower than the value in the data. This shows that disentangling conditional variance and jump intensity is of first-order importance when it comes to breaking up the almost perfect relation between level and slope.

We now change the parameters of the process $\sigma^{2}$. Our objective is to increase the risk premiums by increasing the fundamental amount of risk in the economy. To do so, we increase the persistence of $\sigma^{2}$ by lowering $\kappa_{\sigma}$. We also slightly lower $\sigma_{\sigma}$ and increase the expected jump size, i.e., we replace diffusion risk by jump risk. As a result, the risk premiums increase significantly. The equity risk premium even overshoots the value in the data by a substantial amount. In terms of the option-related moments we find a only a weak relation between the level and slope of the smile, as measured by the $R^{2}$ of the spline fit. We also observe a significant increase in predictability of returns 
via price-dividend ratios and the variance risk premium.

In the next step, we adjust the dynamics of the process $\bar{\sigma}$. Increasing $\kappa_{\bar{\sigma}}$ results in a slight decrease in the equity risk premium, which is, however, still too high when compared to the data. For the option prices we still observe the low relationship between the level and the slope of the IV smile, and the quantiles for $R^{2}$ now bracket the value observed in the data.

In our final step we adjust the parameters for the intensity process $\alpha$ by changing $\kappa_{\alpha, \bar{\sigma}}$ and $\sigma_{\alpha}$, and the expected jump size. This results in our own "Full Model".

\subsection{Model versus Data}

\subsubsection{Basic Cash Flow and Asset Pricing Moments}

In this and the following sections we compare the output of the model to the data. We look at basic cash flow and asset pricing moments, at predictive regressions, and finally at the properties of option prices or, more precisely, implied volatilities. Our focus is on the impact of our two main specification choices, the introduction of a separate intensity driver $\alpha$ and the SQR specification for $\bar{\sigma}^{2}$.

A fundamental requirement for a model specification to be considered basically sensible is that it is able to match the characteristics of the economic fundamentals, in our case consumption and dividends, reasonably well. Table 5 provides a comparison of the data and the different versions of our model. It is obvious that these moments are very similar across all four alternative specifications. This means that one could not distinguish between these different models by just looking at the time-series characteristics of the cash flow variables. Furthermore, the descriptive statistics from the models are also generally close to the data, and the confidence bands generated by the model simulations contain the empirical moments.

At the next level it is of interest whether our model can reproduce stylized facts about the 
equity premium, the risk-free rate, and the variance risk premium. Table 6 shows information on these quantities. Generally speaking, all the models match the first two moments of these quantities equally well and thus properly reproduce the basic asset pricing facts from the data. The confidence intervals for the equity premium are similar across models, ranging roughly from 4 to $10 \%$. The riskfree rate is uniformly low, and the variance risk premium is slightly below 100 bps. As for the cash flow moments the four alternative models are quite similar in their explanatory power, so that the differences in specification do not have an impact at this rather fundamental level. In other words, given the output of the models in terms of the basic asset pricing moments, it would not be possible to say which specification had been used, i.e., with or without the intensity driver $\alpha$ and with an OU or an SQR process for the mean reversion level of the conditional variance.

\subsubsection{Predictability}

In our discussion of the predictability of future excess returns via the current price-dividend ratio we focus on the impact of the dynamics for the mean reversion level of the conditional variance, $\bar{\sigma}^{2}$, which either follows an OU or an SQR process. We had argued above that modeling $\bar{\sigma}^{2}$ with its own square-root in the diffusion component matters beyond the purely technical aspect of guaranteeing positivity. Equation (12) shows that the expected excess return on the dividend claim depends (among other things) on the conditional covariances of the return with the state variables. In case of an OU process the conditional covariance with $\bar{\sigma}^{2}$ is constant, while it does depend on the level of $\bar{\sigma}^{2}$ when this variable follows an SQR process. This implies that future excess returns and the current p-d

ratio have one more component in common than in the OU case. When we now regress realized future excess returns (i.e., expected excess returns plus unpredictable noise) on the current level of the p-d ratio, we would expect the $R^{2}$ to be higher for the SQR specification compared to the OU case. 
The results are shown in Panel A of Table 7, and they are exactly in line with our theoretical considerations. When $\bar{\sigma}^{2}$ follows an SQR process the model-generated $R^{2}$ values are higher on average than for the otherwise identical model with an OU specification. The mean $R^{2}$ for the 5-year horizon increases from 0.14 for the benchmark model to 0.21 for the case without $\alpha$, but with SQR. A similar increase is observed when we move from the model with a separate $\alpha$-process and an OU specification to the full model. Of course, separating intensity and variance in general reduces the $R^{2}$ of the predictive regressions, since there is more independent variation on the two sides of the regression equation, but the full model still matches the data reasonably well and produces results very close to the benchmark specification.

Panels $\mathrm{B}$ and $\mathrm{C}$ in Table 7 contain the results of predictive regressions for future consumption and dividend growth, respectively. As shown by Beeler and Campbell (2012) LRR models tend to exhibit too much predictive power of the p-d ratio for future cahs flow growth, i.e., the average regression coefficients and $R^{2}$ are too high compared to the data. The models we discuss here are no exception with respect to this, but in most cases the confidence bands still cover the values from the data.

Finally, in terms of excess return prediction via the variance risk premium (Table 8) our preferred specification generates slightly lower $R^{2}$ values than the benchmark model, although the confidence bands for our model still include the values from the data for the one and five month horizons.

\subsubsection{Option Prices}

The results for quantities related to option prices are presented in Tables 9 to 12 for maturities of one, three, six, and twelve months, respectively. We will mostly focus on the shortest maturity and 
take the findings for longer maturities as additional robustness checks for our model. The quantities we are interested in most are of course the level $\nu$ and the slope $s$ of the IV smile and their joint dynamics.

In terms of describing the overall level of IV and its changes all four models do a good job. The means of the simulated values are close to the empirically observed average IV for ATM options of $20 \%$, and the confidence bands safely cover the values from the data. Also the volatility of $\nu$ is captured well by all four models. The volatilities of the changes $\Delta \nu$ are somewhat on the low side in the models relative to the data, but overall the properties of the IV level are represented pretty well.

The slope of the smile is computed as the difference between the implied volatility of an OTM put option with a moneyness of 0.9 and that of an ATM option with moneyness equal to 1 . Overall, the models clearly imply that there is a substantial difference in implied volatilities between OTM and ATM options which is in the order of 50 bps. So the model-implied IV smile is significantly skewed, albeit slightly less than in the data, with very similar results across the four different specifications.

The fact that the model-generated slope of the IV smile is slightly lower than in the data is to be expected and should not come as a surprise. Equilibrium models of the type discussed here leave out certain nonstandard factors, which have been shown to be empirically relevant for implied volatilities on index option markets. For example, Bollen and Whaley (2004) show that the excess demand especially for out-of-the-money put options can induce a significant upward move in their implied volatilities. Similarly, in their theoretical model Gârleanu, Pedersen, and Poteshman (2009) show that the demand for options is an important determinant of their prices and IVs, with the effect being especially pronounced for out-of-the-money options. Since the microstructure of the options market is not an element of our model, the output of the model rather represents the (lower) 'fundamental' IV, which is solely due to the presence of risk factors in the economy, than the 'total' 
IV, which is the sum of fundamental and microstructure-induced components. With this in mind, one can summarize that all the models considered here do a good job in terms of matching the IV level and slope.

We now turn to the joint dynamics of level and slope. In the light of the very similar behavior of the models up to now, the differences here are striking. We start by looking at the correlation for the absolute values (i.e., not changes) of level and slope. Here one can immediately see that the independent intensity models match the stylized facts in the data much better than their counterparts with jump intensities, which are an affine function of the conditional variance. They generate a mean correlation of about zero with confidence bands safely covering the correlation of -0.35 estimated from the data. In contrast to this the models with proportional intensity produce correlations that are way too high with values exceeding 0.42 and confidence bands not even reaching zero.

The same is true when we look at options with longer times to maturity. The correlations in the data are somewhat lower in absolute value $(-0.15,-0.08$, and -0.05 for three, six, and twelve months to maturity, respectively). The relative performance of the different models is similar to that for the shortest maturity, with the independent intensity models generating much lower point estimates and confidence bands which clearly contain the estimates from the data, and the affine intensity models failing to reproduce this stylized fact with correlations that are way too high.

Similar conclusions can be drawn for the changes in level and the slope. All the models reproduce the average change of about zero observed in the data for level and the slope pretty well. However, just like before, only the models with a separate intensity driver can match the empirically observed negative value for the correlation. In the data the correlation between monthto-month changes in level and slope is negative ${ }^{23}$ with a value of -0.16 for the options with one

\footnotetext{
${ }^{23}$ The negative correlations between level and slope and also between the changes in the two variables are not special to our monthly observation frequency. The corresponding numbers for the two correlations in the data are -0.296 and -0.295 for daily and -0.285 and -0.038 for weekly observation frequency.
} 
month to maturity, and the average from the simulations for the full model is -0.228 . Also the second specification with a separate intensity process $\alpha$ performs well here, albeit with a mean that is further away from the value in the data. The difference to the models with proportional intensity is again very pronounced. The average correlation in the simulations is above 0.66 , and the $90 \%$ confidence bands are also far away from zero, let alone from negative values.

As mentioned already in the introduction the key point here is not that it would be generally impossible for affine intensity models to produce a negative correlation between level and slope. It is rather the tight link between level and slope, irrespective of whether the functional form is linear or not. To allow for a basically arbitrary link between level and slope we consider a robust nonparametric spline function relating level and slope as plotted in Figure $1 .{ }^{24}$

Table 9 shows that in the data the spline produces an $R^{2}$ of 0.22 based on options with one month to maturity. Models with separate intensity processes are able to match this value rather closely with confidence intervals safely including the value from the data. Again models without the $\alpha$-process generate a goodness of fit that is way too high with average $R^{2}$ values of 0.6 or higher. The benchmark model is rather extreme in this regard and basically predicts a perfect fit between slope and level.

This can also be seen from Figure 2, where the upper and the lower left graphs represent the spline functions for the models with a separate intensity process, while the two graphs on the right show the corresponding pictures for the affine intensity specifications. The fit to the curve is much tighter for the latter than for the former. From the numbers in Tables 10 to 12 one can see that the results for short-term options also carry over to longer maturities. The models with affine intensity continue to produce goodness of fit measures which are significantly greater than in the data.

\footnotetext{
${ }^{24}$ The details of the spline fitting procedure are described in Online Appendix D. The graphs for three, six, and twelve months to maturity look very similar and are available from the authors upon request.
} 
The same conclusions arise when we look at the increments in level and slope. The results in Table 9 show that for the one month maturity options the $R^{2}$ in the data is 0.09 . Again, only the models with a separate intensity process are able to match this value closely. Tables 10 to 12 show that these results are also obtained for longer option maturities.

Overall, the empirical evidence on the dynamics of the two easily observable characteristics of the IV smile, its level and its slope (as well as their changes), provides a strong case for a separate process driving the intensity of jumps in the context of a long-run risk model.

\subsection{Decomposing the Equity Premium}

The general topic of our paper is to disentangle the role of jump risk and volatility risk in our LRR model. The starting point of our argument was that jump and diffusive risks represent two fundamentally different types of risk, and that is also why it is of natural interest to see their respective contributions to the risk premia in the model.

This decomposition of a risk premium can be done in (at least) two ways. First, one can be interested in the shares of diffusive and jump-related premia in the total equity risk premium and how these shares can then be attributed in a second step to the stochastic variables in the model, i.e., to $x, \sigma^{2}, \alpha$ (if included), $\bar{\sigma}^{2}$, and $\ln (D)$.

The results for this type of decomposition are shown in Table 13. As an example of how to read the entries in these tables consider Panel A for the full model in Table 13. The total equity risk premium here is $6.078 \%$, with $1.952 \%$ coming from diffusive and $4.126 \%$ from jump risk (see column 'Sum'). ${ }^{25}$ The $1.952 \%$ diffusive premium is composed of $1.125 \%$ coming from the fact that $x$ is stochastic, of $0.156 \%$ from stochastic variation in $\sigma^{2}$, and so on. The jump-related components

\footnotetext{
${ }^{25}$ Theoretically the total equity risk premium shown in Table 13 should be equal to the numbers shown in Table 6 . The minor differences are due to simulation error.
} 
are to be interpreted analogously, where we look at the contributions of jumps in $x, \sigma^{2}$, and $\alpha$.

Second, one may want to know how the equity risk premium depends on the current values of the state variables capturing uncertainty in the model, i.e., of $\sigma^{2}, \alpha$ (where present), and $\bar{\sigma}^{2}$. The results are shown in Table 14. To give again an idea how to read the entries of these tables, consider Panel A for the full model. The equation for the total equity risk premium $E R P_{t}$ at time $t$ in this model would be $E R P_{t}=-0.396+1.306 \sigma_{t}^{2}+4.129 \alpha_{t}+1.039 \bar{\sigma}_{t}^{2}$, where the diffusive part is given by $-0.396+1.306 \sigma_{t}^{2}+0.003 \alpha_{t}+1.039 \bar{\sigma}_{t}^{2}$, and the jump part comes exclusively from $\alpha$ with a coefficient of 4.126. Since all the variables have a stationary mean equal to 1 , these sums are on average equal to $1.952 \%$ and $4.126 \%$, respectively.

Looking at Table 13 first, one can see that for all model specifications the share of the diffusive part of the equity premium is in between 30 and 50 percent, so that the jump part is consistently somewhat more important. For the diffusive part of the equity premium the most important drivers are $x$ and $\bar{\sigma}^{2}$, i.e., the processes with the lower mean reversion speeds, again a characteristic which is shared by all versions of the model. Note that the negative contribution of $\ln (D)$ to the diffusive part of the equity premium in all versions of the model is due to the Jensen correction term in Equation equation (12), so it is merely technical in nature.

With respect to jump risk, one can also identify two important fundamental drivers, namely the stochastic growth rate $x$ and the process $\sigma$. In all four versions of the model they account for almost all of the jump-related equity risk premium. This implies that investors are very concerned about adverse changes in growth and an increase in the conditional variance.

Table 14 shows the impact of the fundamental risk sources $\sigma^{2}, \alpha$, and $\bar{\sigma}^{2}$ on the conditional equity risk premium, which allows us to attribute changes in the equity risk premium to changes in normal diffusion risk, in the jump intensity, and in the long-run level of uncertainty. In the full model 
(Panel A) the amount of diffusive risk $\sigma^{2}$ has a coefficient of 1.306 , while the amount of jump risk $\alpha$ is much more important with a coefficient of 4.129. The long-run level of uncertainty $\bar{\sigma}^{2}$ enters with a coefficient of 1.039 .

In an otherwise identical model, where the jump intensity is affine in $\sigma^{2}$ (Panel B), the coefficient of $\sigma^{2}$, which now drives the quantities of both diffusive and jump risk, is 5.208, i.e., roughly equal to the sum of the coefficients for $\sigma^{2}$ and $\alpha$ in Panel A. Only in the full model it is possible to tell separately how the equity premium reacts to a variation in short-term diffusive variance and to a change in the jump intensity, and it becomes obvious that the equity premium reacts in a much stronger fashion to a marginal change in the jump intensity than to a marginal change in diffusive variance.

\subsection{Comparative Static Analysis of Jumps}

In this section we analyze the impact of the jump component in more detail. To this end we use a comparative static analysis where we successively introduce the jump component into the dynamics of the state variables $x, \sigma$, and $\alpha$. We use our model calibration for the full model shown in Table 2 and present the results of this exercise in Table 15.

The columns in panel A of Table 15 show the results of the model simulation when we shut down all jump processes by setting the parameter $l_{i, 1}$ in equation (7) equal to zero for all $i \in\{x, \sigma, \alpha\} .{ }^{26}$ The remaining panels in Table 15 show the results for setting the parameters $l_{i, 1}$ successively equal to the value in the full model.

The first interesting and somewhat surprising result is that the effects are hardly noticeable when looking at the moments for dividend and consumption growth. In terms of asset pricing mo-

\footnotetext{
${ }^{26}$ Since we are using our "Full Model" specification, we have $\varphi_{\alpha}=1$.
} 
ments, however, we see large differences across the model specifications. First, in the case of no jumps the equity risk premium is less than $1 \%$, and its volatility is too low. ${ }^{27}$ Furthermore, the variance risk premium all but disappears.

Concerning the predictability results, we see that the jump components generally only have a limited influence on the return predictability using the price-dividend ratio. It is much more interesting, however, to look at the predictability using the variance risk premium when we introduce jumps into the intensity process, i.e., in the full model, as compared to only adding jumps to the long-run mean and the variance processes. We observe that for the one-month horizon the $R^{2}$ is now much closer to the data than for the other specifications. Furthermore the term structure of $R^{2}$ is no longer monotonically increasing as before, but it now tends to be lower for longer horizons, just like in the data.

The most interesting aspect of this exercise is the impact of the jump components on the option-related moments. The level and slope of the volatility smile increase when the number of potentially jumping state variables increases, and the same is true for the variation of these moments. But most importantly, the relation between the level and slope of the smile also depends on the number of state variables that jump. Without jumps, $\alpha$ no longer has an impact, and the spline fit gives an $R^{2}$ of more than 0.99 . The correlation between level and slope is also large with values between 0.5 and 0.95 . Introducing jumps with an intensity driven by a process other than variance breaks this tight relation. The introduction of jumps in $x$ and $\sigma$ reduces the $R^{2}$ from 0.996 to 0.501 and 0.684 , respectively. Additional jumps in $\alpha$ brings the $R^{2}$ to around 0.25 , which is close to the value found in the data.

\footnotetext{
${ }^{27}$ Note that the result is not comparable to the analysis in Section 5.3 since the change in the vector $l_{1}$ affects the overall equilibrium solution.
} 


\section{Conclusion}

In this paper we propose an LRR model with stochastic volatility with a time-varying mean reversion level and jumps in the state variables. The special feature of our model is that, instead of making the jump intensity an affine function of the conditional variance, we introduce a separate process for it.

The motivation for our setup is straightforward. In a model, where the jump intensity is an affine function of the conditional variance, the level and the slope of the IV smile would be perfectly correlated. In the data, however, we observe only a weak link between these two quantities, so that jumps and diffusions represent two distinctive sources of risk.

While models both with and without a separate intensity process are able to match the standard asset pricing moments like the equity premium, the risk-free rate, the variance risk premium, and basic option pricing data, only the specifications with this extra process for the jump intensity are able to reproduce the dynamic relationship between the easily observable quantities level and slope as it shows up in the data. Overall our results provide a strong case for including a separate intensity process in equilibrium jump-diffusion models.

\section{References}

Backus, D., M. Chernov, and I. Martin, 2011, Disasters Implied by Equity Index Options, Journal of Finance 66, 1969-2012.

Bansal, R., R. Dittmar, and C. Lundblad, 2005, Consumption, Dividends, and the Cross-Section of Equity Returns, Journal of Finance 60, 1639-1672.

Bansal, R., R. Gallant, and G. Tauchen, 2007, Rational Pessimism, Rational Exuberance, and Asset Pricing Models, Review of Econimic Studies 74, 1005-1033. 
Bansal, R., D. Kiku, and A. Yaron, 2012, Risks for the Long Run: Estimation with Time Aggregation, NBER Working Paper No. 18305.

Bansal, R., and I. Shaliastovich, 2011, Learning and Asset-Price Jumps, Review of Financial Studies $24,2738-2780$.

Bansal, R., and I. Shaliastovich, 2013, A Long-Run Risks Explanation of Predictability Puzzles in Bond and Currency Markets, Review of Financial Studies 26, 1-33.

Bansal, R., and A. Yaron, 2004, Risks For the Long Run: A Potential Resolution of Asset Pricing Puzzles, Journal of Finance 59, 1481-1509.

Barro, R.J., 2009, Rare Disasters, Asset Prices, and Welfare Costs, American Economic Review 99, $243-264$.

Bates, D., 2000, Post-'87 crash fears in the S\&P 500 futures options market, Journal of Econometrics 94, 181-238.

Beeler, J., and J. Y. Campbell, 2012, The Long-Run Risks Model and Aggregate Asset Prices: An Empirical Assessment, Critical Finance Review 1, 141-182.

Benzoni, L., P. Collin-Dufresne, and R. S. Goldstein, 2011, Explaining Asset Pricing Puzzles Associated with the 1987 Market Crash, Journal of Financial Economics 101, 552-573.

Bloom, Nicholas, 2009, The impact of uncertainty shocks, Econometrica 77, 623-685.

Bollen, N., and R. Whaley, 2004, Does Net Buying Pressure Affect the Shape of Implied Volatility Functions?, Journal of Finance 59, 711-753.

Bollerslev, T., N. Sizova, and G. Tauchen, 2012, Volatility in Equilibrium: Asymmetries and Dynamic Dependencies, Review of Finance 16, 31-80. 
Bollerslev, T., G. Tauchen, and H. Zhou, 2010, Expected Stock Returns and Variance Risk Premia, Review of Financial Studies 22, 4463-4492.

Campbell, J.Y., and R. J. Shiller, 1988, Stock Prices, Earnings, and Expected Dividends, Journal of Finance 43, 661-676.

Carr, P., and L. Wu, 2009, Variance Risk Premiums, Review of Financial Studies 22, 1311-1341.

Christoffersen, P., B. Feunou, Y. Jeon, and C. Ornthanalai, 2016, Time-Varying Crash Risk: The Role of Stock Market Liquidity, SSRN Working Paper.

Christoffersen, P., K. Jacobs, and C. Ornthanalai, 2012, Dynamic jump intensities and risk premiums: Evidence from S\&P 500 returns and options, Journal of Financial Economics 106, 447-472.

Colacito, R., and M. M. Croce, 2011, Risks for the Long Run and the Real Exchange Rate, Journal of Political Economy 119, 153-181.

Constantinides, G. M., and A. Ghosh, 2011, Asset Pricing Tests with Long-run Risks in Consumption Growth, Review of Asset Pricing Studies 1, 96-136.

Cremers, M., M. Halling, and D. Weinbaum, 2015, Aggregate Jump and Volatility Risk in the CrossSection of Stock Returns, Journal of Finance 70, 577-614.

Drechsler, I., and A. Yaron, 2011, What's Vol Got to Do With It, Review of Financial Studies 24, $1-45$.

Duffie, D., J. Pan, and K. Singleton, 2002, Transform Analysis and Asset Pricing for Affine Jumpdiffusions, Econometrica 68, 1343-1376.

Egloff, D., M. Leippold, and L. Wu, 2010, The Term Structure of Variance Swap Rates and Optimal Variance Swap Investments, Journal of Financial and Quantitative Analysis 45, 1279-1310. 
Epstein, L. G., and S. E. Zin, 1989, Substitution, Risk Aversion, and the Temporal Behavior of Consumption Growth and Asset Returns I: A Theoretical Framework, Econometrica 57, 937-969.

Eraker, B., and I. Shaliastovich, 2008, An Equilibrium Guide to Designing Affine Pricing Models, Mathematical Finance 18, 519-543.

Foresi, S., and L. Wu, 2005, Crash-O-Phobia: A Domestic Fear or a Worldwide Concern?, Journal of Derivatives 13, 8-21.

Gârleanu, N., L. Pedersen, and A. Poteshman, 2009, Demand-Based Option Pricing, Review of Financial Studies 22, 4259-4299.

Hansen, L. P., J. C. Heaton, and N. Li, 2008, Consumption Strikes Back? Measuring Long-Run Risk, Journal of Political Economy 116, 260-302.

Hasseltoft, H., 2012, Stocks, Bonds, and Long-Run Consumption Risks, Journal of Financial and Quantitative Analysis 47, 309-332.

Jagannathan, R., and S. Marakani, 2011, Long Run Risks \& Price/Dividend Ratio Factors, NBER Working Paper No. 17484.

Juillard, C., and A. Ghosh, 2012, Can Rare Events Explain the Equity Premium Puzzle, Review of Financial Studies 25, 3037-3076.

Jurado, K., S.C. Ludvigson, and S. Ng, 2015, Measuring Uncertainty, American Economic Review 105, 1177-1216.

Kaltenbrunner, G., and L. A. Lochstoer, 2010, Long-Run Risk through Consumption Smoothing, Review of Financial Studies 23, 3190-3224.

Kiku, D., 2006, Is the Value Premium a Puzzle?, Working Paper. 
Malloy, C., T. Moskowitz, and A. Vissing-Jorgensen, 2009, Long-Run Stockholder Consumption Risk and Asset Returns, Journal of Finance 64, 2427-2479.

McCracken, M. W., and S. Ng, 2015, FRED-MD: A Monthly Database for Macroeconomic Research, forthcoming Journal of Business 6 Economic Statistics.

Pan, J., 2002, The jump-risk premia implicit in options: evidence from an integrated time-series study, Journal of Financial Economics 63, 3-50.

Pástor, L., and R.F. Stambaugh, 2003, Liquidity risk and expected stock returns, Journal of Political Economy 111, 642-685.

Rietz, T.E., 1988, The equity premium: A solution, Journal of Monetary Economics 22, 117-131.

Santa-Clara, P., and S. Yan, 2010, Crashes, Volatility, and the Equity Premium: Lessons from S\&P 500 Options, Review of Economics and Statistics 92, 435-451.

Shaliastovich, Ivan, 2015, Learning, Confidence, and Option Prices, Journal of Econometrics 187, 18âĂŞ42.

Todorov, V., 2010, Variance Risk Premium Dynamics, Review of Financial Studies 23, 345-383.

Yan, S., 2011, Jump risk, stock returns, and slope of implied volatility smile, Journal of Financial Economics 99, 216-233.

Zhou, G., and Y. Zhu, 2012, A Long-run Risks Model with Long- and Short-run Volatilities: Explaining Predictability and Volatility Risk Premium, Working Paper.

Zhou, G., and Y. Zhu, 2015, Macroeconomic Volatilities and Long-run Risks of Asset Prices, Management Science 61, 413-430. 
Table 1

Level and Slope versus Macro Variables

\begin{tabular}{l|r|r}
\hline Regressor & Level & Slope \\
\hline Constant & -10.79 & 0.1202 \\
& $(-1.70)$ & $(5.25)$ \\
\hline Output and income & 0.0690 & -0.0125 \\
& $(4.32)$ & $(-2.13)$ \\
\hline Labor market & 0.0213 & -0.0187 \\
& $(0.84)$ & $(-1.76)$ \\
\hline Consumption and housing & -0.0807 & 0.0081 \\
& $(-9.28)$ & $(2.25)$ \\
\hline Orders and inventories & 0.0001 & 0.0142 \\
& $(0.01)$ & $(1.40)$ \\
\hline Money and credit & -0.0106 & 0.0082 \\
& $(-0.70)$ & $(1.34)$ \\
\hline Interest rate and exchange rates & -0.0511 & 0.0120 \\
& $(-3.50)$ & $(1.99)$ \\
\hline Prices & -0.1492 & -0.0086 \\
& $(-3.44)$ & $(-0.57)$ \\
\hline Stock Market & -0.0114 & 0.0018 \\
& $(-2.17)$ & $(0.93)$ \\
\hline JLN & 0.3786 & -0.0363 \\
& $(4.85)$ & $(-1.27)$ \\
\hline PS & -0.2492 & -0.0088 \\
& $(-2.92)$ & $(-0.31)$ \\
\hline adj. $R^{2}$ & 0.6326 & 0.1396 \\
\hline
\end{tabular}

The table shows the estimated coefficients, associated Newey-West $t$-statistics (in parentheses), and adjusted $R^{2}$ of a regression of our monthly measure for the level and the slope of the implied volatility smile for options on the $\mathrm{S} \& \mathrm{P}$ 500 index on the standardized first principal components of the eight group of macro variables (recorded at a monthly frequency) presented in McCracken and $\mathrm{Ng}$ (2015) as well as on JLN, the macro uncertainty factor from Jurado, Ludvigson, and $\mathrm{Ng}$ (2015) (for $h=1$ in their notation). and PS, the liquidity factor from Pástor and Stambaugh (2003). The left column shows the name of the respective group of macro variables. Options data are taken from OptionMetrics. We use only data on put options with a trading volume greater than 100 contracts. The level of the implied volatility smile is defined as the IV of an option with 30 days to maturity and a moneyness (defined as the ratio of strike price to index price) of one. The slope $s$ is given by the difference between the IVs of a 30-day option with a moneyness of 0.9 and the level of the IV smile. The implied volatility for options with exactly 30 days to maturity and exact moneyness levels of one and 0.9 are found by interpolation. The time span for the options data is from January 1996 to August 2015. 
Table 2

Model Parameters

\begin{tabular}{|c|c|c|c|c|}
\hline & A: Full Model & B: Without $\alpha, \mathbf{S Q R}$ & C: With $\alpha$, OU & $\begin{array}{l}\text { D: Without } \alpha, \text { OU } \\
\text { (benchmark model) }\end{array}$ \\
\hline & \multicolumn{4}{|c|}{ Investor } \\
\hline$\gamma$ & 8.800 & 10.000 & 8.800 & 10.000 \\
\hline$\psi$ & 2.000 & 2.000 & 2.000 & 2.000 \\
\hline$\beta$ & \multicolumn{3}{|c|}{ Cash Flows } & 0.012 \\
\hline$E(\Delta c)$ & 0.019 & 0.019 & 0.019 & 0.019 \\
\hline$\sigma_{c}$ & 0.023 & 0.023 & 0.023 & 0.023 \\
\hline$w_{c}$ & 0.500 & 0.500 & 0.500 & 0.500 \\
\hline$E(\Delta \delta)$ & 0.019 & 0.019 & 0.019 & 0.019 \\
\hline$\sigma_{\delta}$ & 2.500 & 2.500 & 2.500 & 2.500 \\
\hline$w_{\delta}$ & 0.130 & 0.130 & 0.130 & 0.130 \\
\hline$w_{c \delta}$ & 0.125 & 0.125 & 0.125 & 0.125 \\
\hline \multicolumn{5}{|c|}{ State Variables } \\
\hline$\kappa_{x}$ & 0.288 & 0.288 & 0.288 & 0.288 \\
\hline$\sigma_{x}$ & 0.009 & 0.009 & 0.009 & 0.009 \\
\hline$w_{x}$ & 1.000 & 1.000 & 1.000 & 1.000 \\
\hline$E\left[\xi^{x}\right]$ & 0.000 & 0.000 & 0.000 & 0.000 \\
\hline $\operatorname{Std}\left[\xi^{x}\right]$ & 0.009 & 0.009 & 0.009 & 0.009 \\
\hline$l_{0 x}$ & 0.000 & 0.000 & 0.000 & 0.000 \\
\hline$\hat{l}_{x}$ & 0.800 & 0.800 & 0.800 & 0.800 \\
\hline$\kappa_{\sigma, \bar{\sigma}}$ & 0.450 & 1.560 & 0.450 & 1.560 \\
\hline$\sigma_{\sigma}$ & 1.039 & 1.212 & 1.039 & 1.212 \\
\hline$w_{\sigma}$ & 1.000 & 1.000 & 1.000 & 1.000 \\
\hline$E\left[\xi^{\sigma}\right]$ & 2.750 & 2.550 & 2.750 & 2.550 \\
\hline$l_{0 \sigma}$ & 0.000 & 0.000 & 0.000 & 0.000 \\
\hline$\hat{l}_{\sigma}$ & 0.800 & 0.800 & 0.800 & 0.800 \\
\hline$\kappa_{\alpha, \bar{\sigma}}$ & 2.400 & - & 2.400 & - \\
\hline$\sigma_{\alpha}$ & 0.277 & - & 0.277 & - \\
\hline$w_{\alpha}$ & 1.000 & - & 1.000 & - \\
\hline$E\left[\xi^{\alpha}\right]$ & 2.750 & - & 2.750 & - \\
\hline$l_{0 \alpha}$ & 0.000 & - & 0.000 & - \\
\hline$\hat{l}_{\alpha}$ & 0.800 & - & 0.800 & - \\
\hline$\kappa_{\bar{\sigma}}$ & 0.240 & 0.180 & 0.240 & 0.180 \\
\hline$\sigma_{\bar{\sigma}}$ & 0.346 & 0.346 & 0.346 & 0.346 \\
\hline
\end{tabular}

The table shows the parameters for the different versions of our model. 'Full Model' refers to the specification with an autonomous process $\alpha$ for the stochastic intensity and a square root (SQR) process for the level of mean reversion of the stochastic variance, $\bar{\sigma}^{2}$. The other specifications do not include $\alpha$ ('without $\alpha$ '), and/or $\bar{\sigma}^{2}$ is assumed to follow an Ornstein Uhlenbeck (OU) process. We denote the parameters for which the values in the calibration of the 'Full Model' depart from the values in the benchmark model in bold. All parameters are annualized. 
Table 3

Model Parameters: Details on the Recalibration

\begin{tabular}{|c|c|c|c|c|}
\hline & \multirow[t]{2}{*}{ A: DY Calibration } & B: Change $\sigma$ & C: Change $\bar{\sigma}$ & \multirow[t]{2}{*}{ D: Full Model } \\
\hline & & Inves & & \\
\hline$\gamma$ & 10.000 & 8.800 & 8.800 & 8.800 \\
\hline$\psi$ & 2.000 & 2.000 & 2.000 & 2.000 \\
\hline \multirow[t]{2}{*}{$\beta$} & 0.012 & 0.012 & 0.012 & 0.012 \\
\hline & & Cash $\mathbf{F}$ & & \\
\hline$E(\Delta c)$ & 0.019 & 0.019 & 0.019 & 0.019 \\
\hline$\sigma_{c}$ & 0.023 & 0.023 & 0.023 & 0.023 \\
\hline$w_{c}$ & 0.500 & 0.500 & 0.500 & 0.500 \\
\hline$E(\Delta \delta)$ & 0.019 & 0.019 & 0.019 & 0.019 \\
\hline$\sigma_{\delta}$ & 2.500 & 2.500 & 2.500 & 2.500 \\
\hline$w_{\delta}$ & 0.130 & 0.130 & 0.130 & 0.130 \\
\hline \multirow[t]{2}{*}{$w_{c \delta}$} & 0.125 & 0.125 & 0.125 & 0.125 \\
\hline & & State Va & bles & \\
\hline$\kappa_{x}$ & 0.288 & 0.288 & 0.288 & 0.288 \\
\hline$\sigma_{x}$ & 0.009 & 0.009 & 0.009 & 0.009 \\
\hline$w_{x}$ & 1.000 & 1.000 & 1.000 & 1.000 \\
\hline$E\left[\xi^{x}\right]$ & 0.000 & 0.000 & 0.000 & 0.000 \\
\hline $\operatorname{Std}\left[\xi^{x}\right]$ & 0.009 & 0.009 & 0.009 & 0.009 \\
\hline$l_{0 x}$ & 0.000 & 0.000 & 0.000 & 0.000 \\
\hline$\hat{l}_{x}$ & 0.800 & 0.800 & 0.800 & 0.800 \\
\hline$\kappa_{\sigma, \bar{\sigma}}$ & 1.560 & 0.450 & 0.450 & 0.450 \\
\hline$\sigma_{\sigma}$ & 1.212 & 1.039 & 1.039 & 1.039 \\
\hline$w_{\sigma}$ & 1.000 & 1.000 & 1.000 & 1.000 \\
\hline$E\left[\xi^{\sigma}\right]$ & 2.550 & 2.750 & 2.750 & 2.750 \\
\hline$l_{0 \sigma}$ & 0.000 & 0.000 & 0.000 & 0.000 \\
\hline$\hat{l}_{\sigma}$ & 0.800 & 0.800 & 0.800 & 0.800 \\
\hline$\kappa_{\alpha, \bar{\sigma}}$ & 1.560 & 1.560 & 1.560 & 2.400 \\
\hline$\sigma_{\alpha}$ & 1.212 & 1.212 & 1.212 & 0.277 \\
\hline$w_{\alpha}$ & 1.000 & 1.000 & 1.000 & 1.000 \\
\hline$E\left[\xi^{\alpha}\right]$ & 2.550 & 2.550 & 2.550 & 2.750 \\
\hline$l_{0 \alpha}$ & 0.000 & 0.000 & 0.000 & 0.000 \\
\hline$\hat{l}_{\alpha}$ & 0.800 & 0.800 & 0.800 & 0.800 \\
\hline$\kappa_{\bar{\sigma}}$ & 0.180 & 0.180 & 0.240 & 0.240 \\
\hline$\sigma_{\bar{\sigma}}$ & 0.346 & 0.346 & 0.346 & 0.346 \\
\hline
\end{tabular}

The table shows the parameters for the each step in our calibration exercise. "DY Calibration" refers to the calibration from our benchmark model where we use the parameter values of the $\sigma$ process for the process $\alpha$. "Change $\sigma$ " refers to the step where the parameters for the $\sigma$ process are changed. "Change $\bar{\sigma}$ " shows the parameter values for the calibration with the adjustments in the $\bar{\sigma}$ process. The "Full Model" specification shows the parameters used in the main text. The changes in each step are shown in bold numbers. All parameters are annualized. 


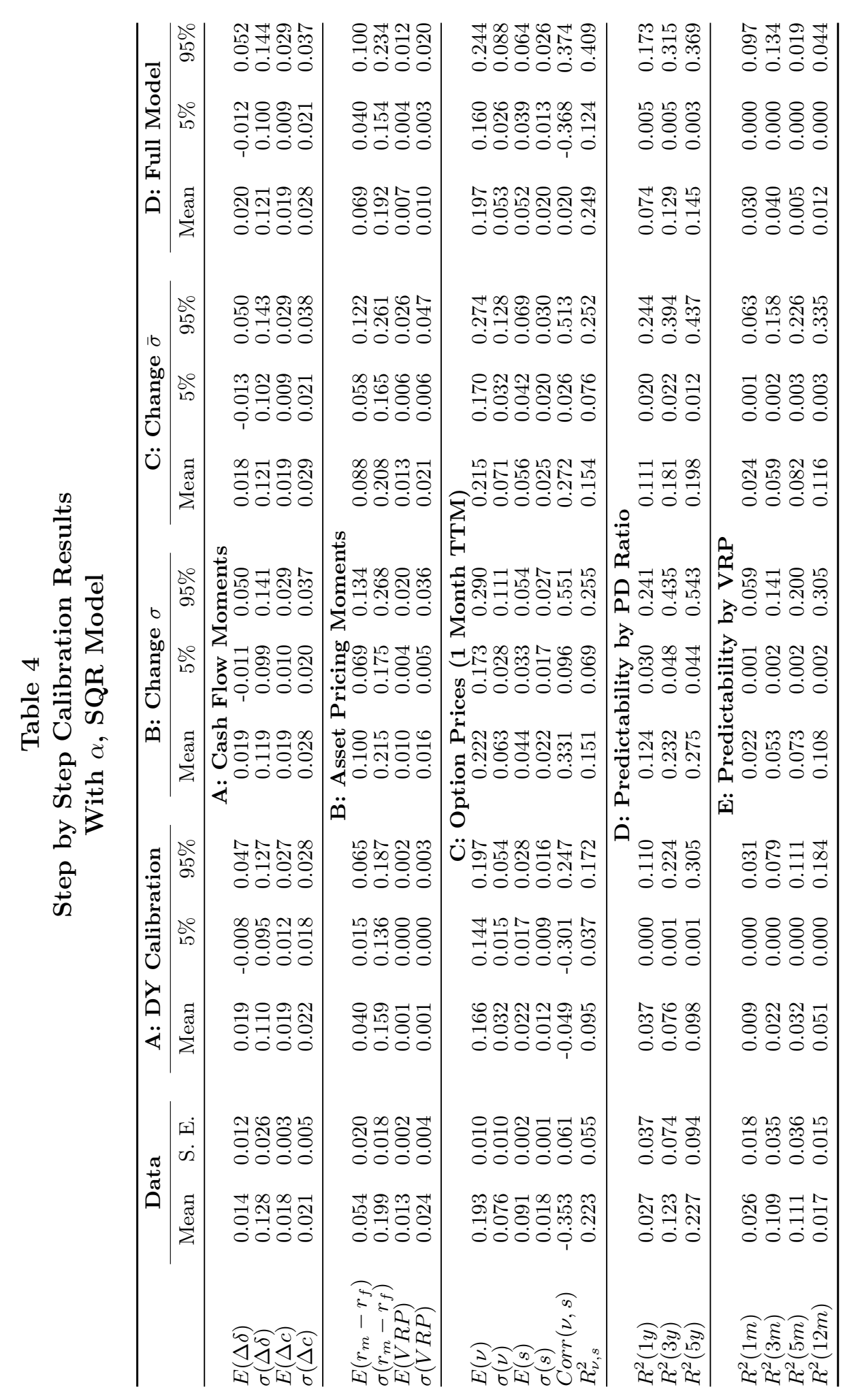

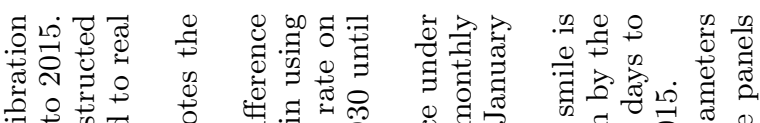

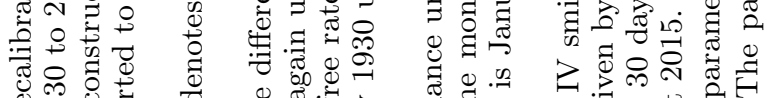

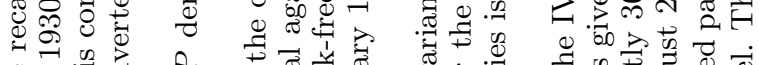

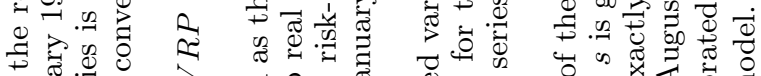

. 光:

هำ

ऊ

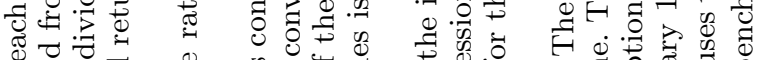

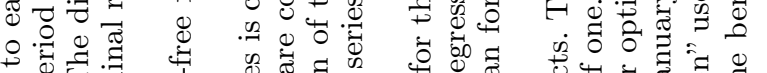

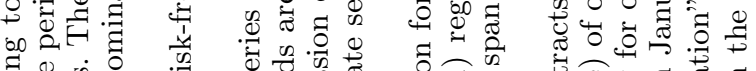

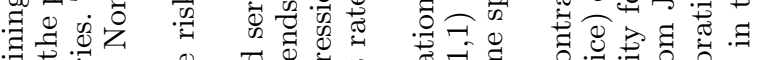

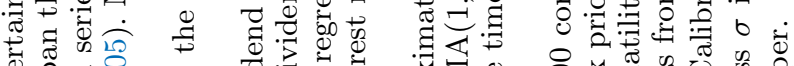

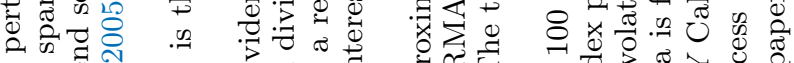

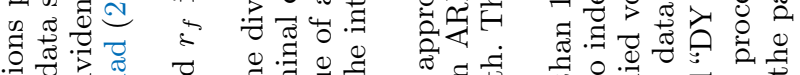

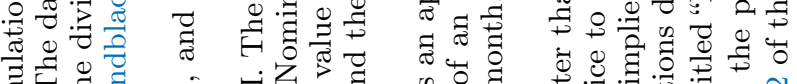

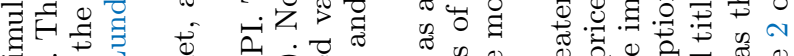

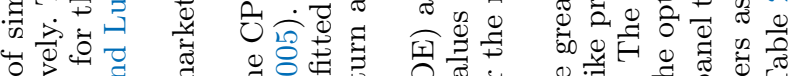

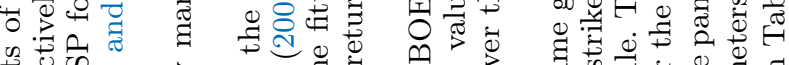

क्षे की

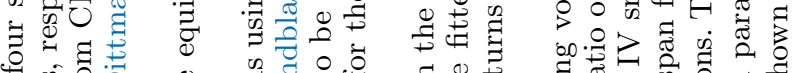

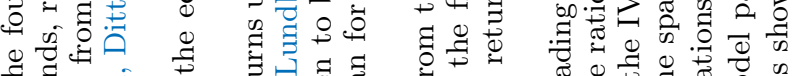

ఫ记

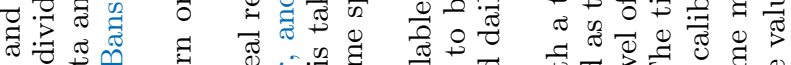

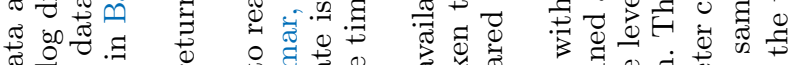

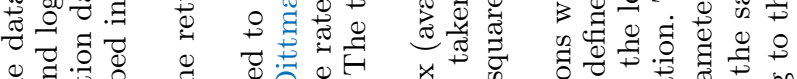

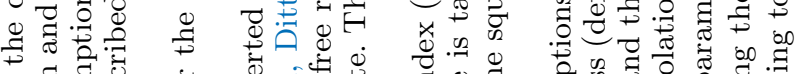

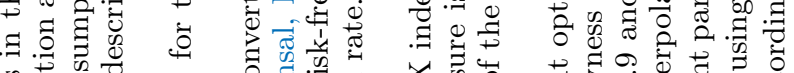

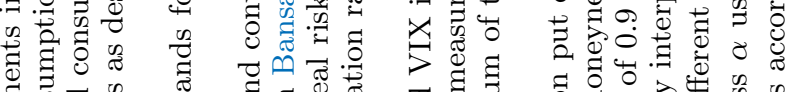

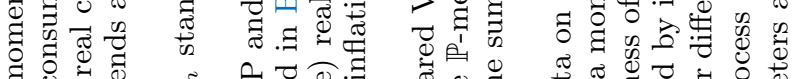

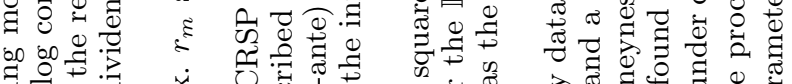

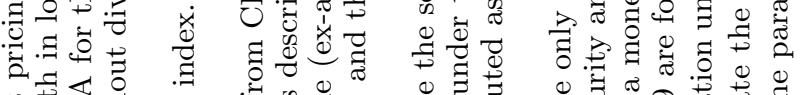

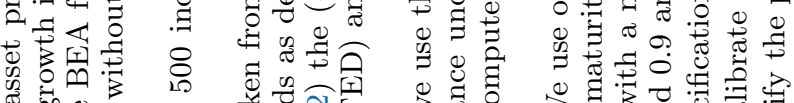

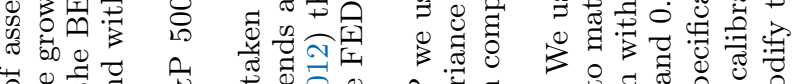

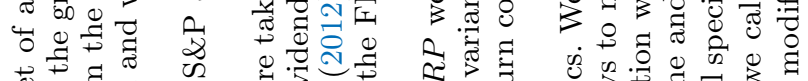

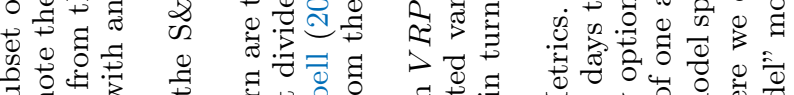

कि

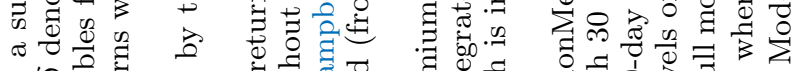

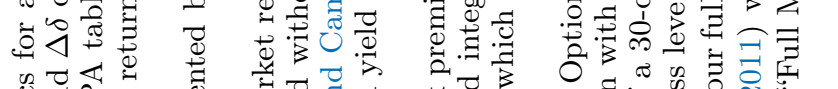

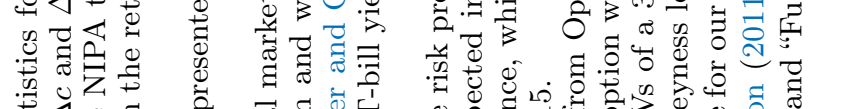

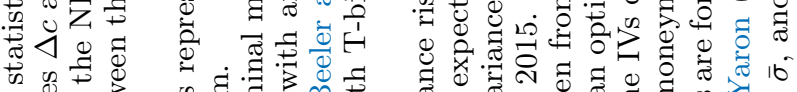

की †.

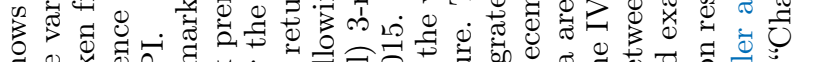

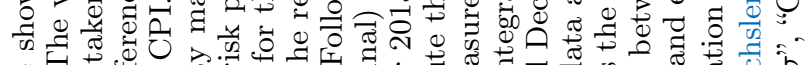

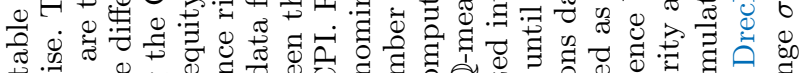

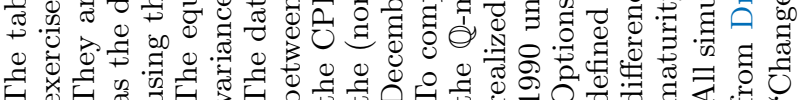




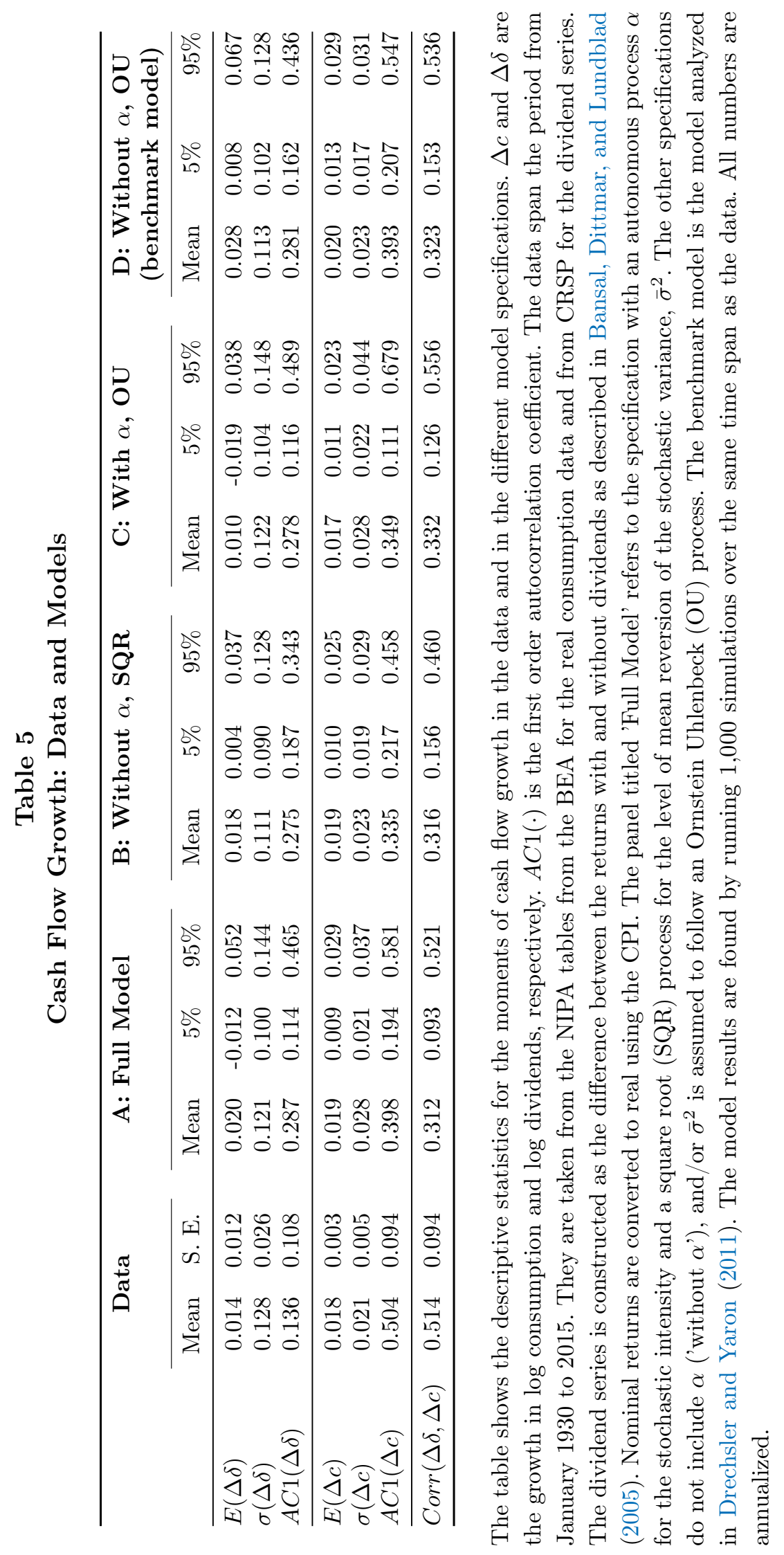




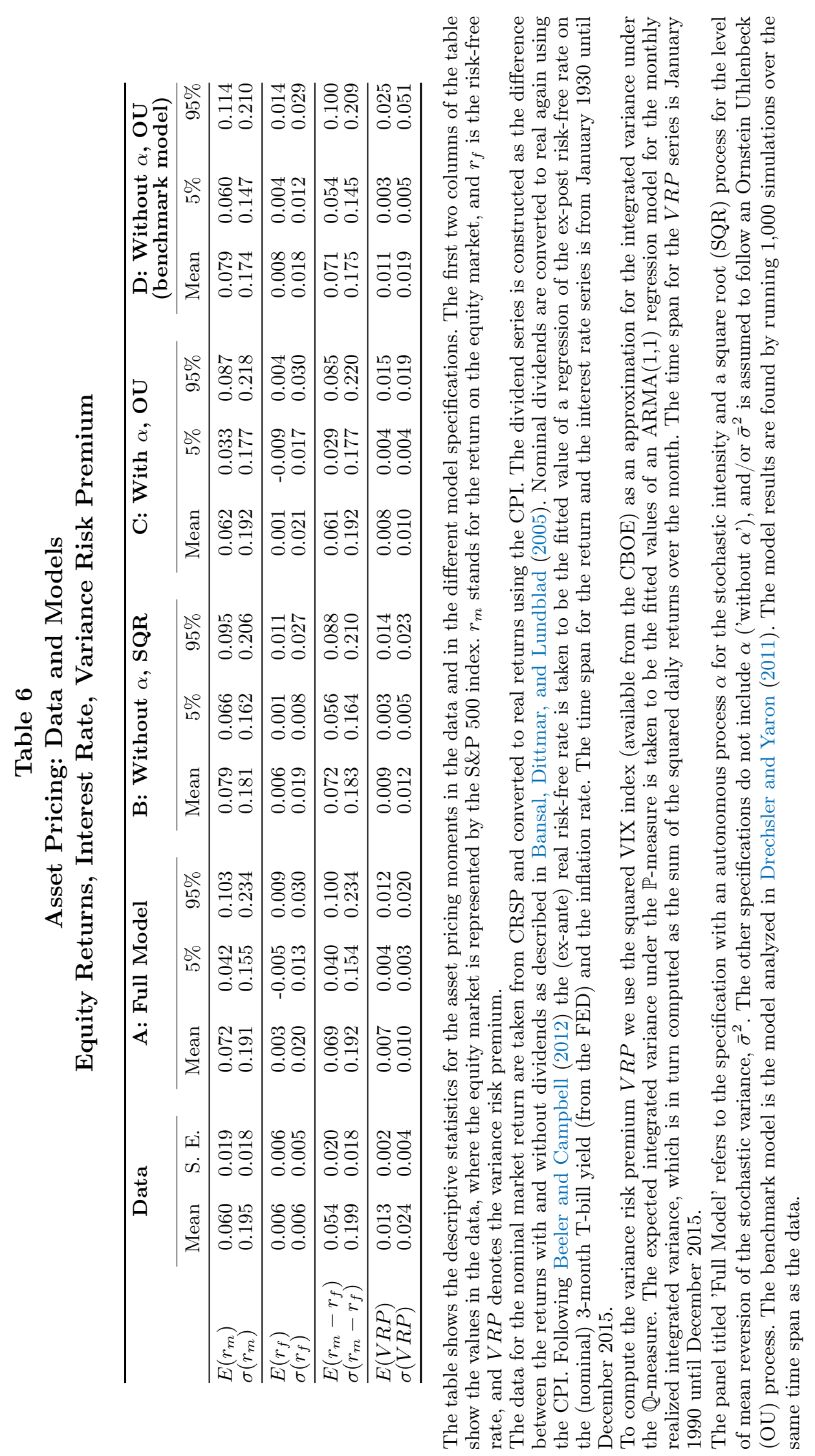




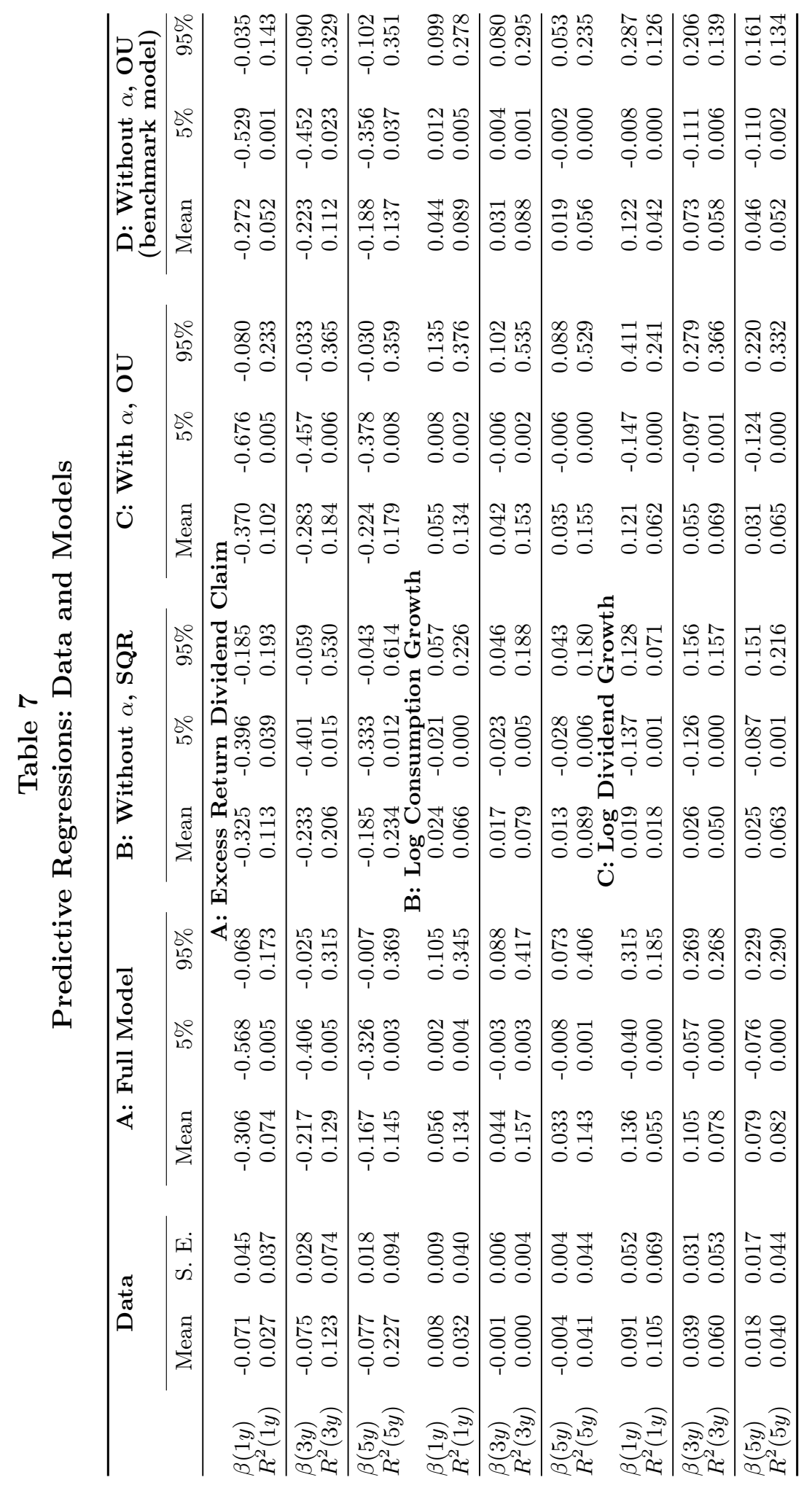

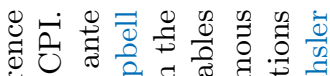

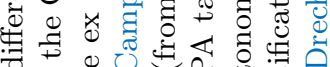

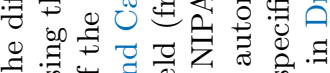

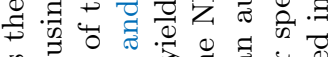

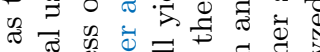

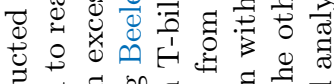
$\exists$.

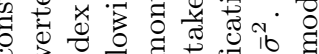

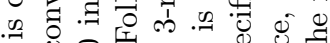

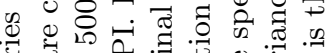
क्ष 0 o 웜 证

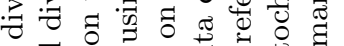

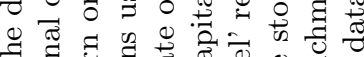

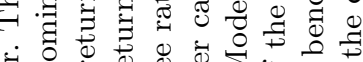

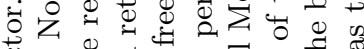

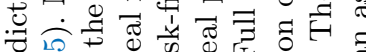

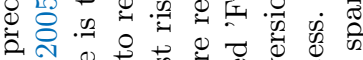

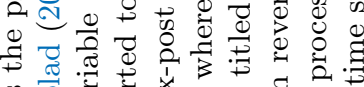
品

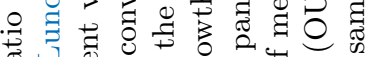
踏 궁 ส

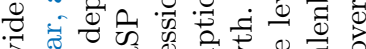

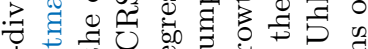
d

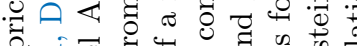

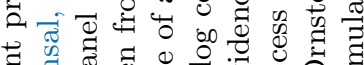
诺 의

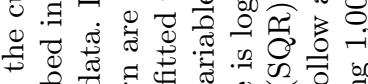
의

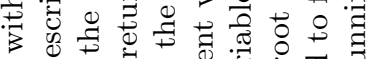

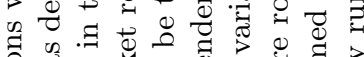
의 过

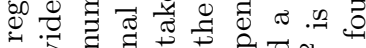

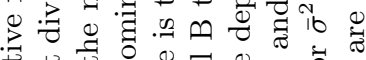

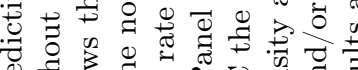

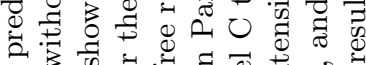
의

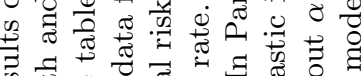

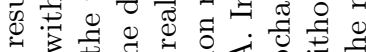
和出 o o ज.

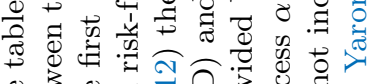

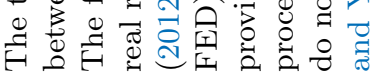




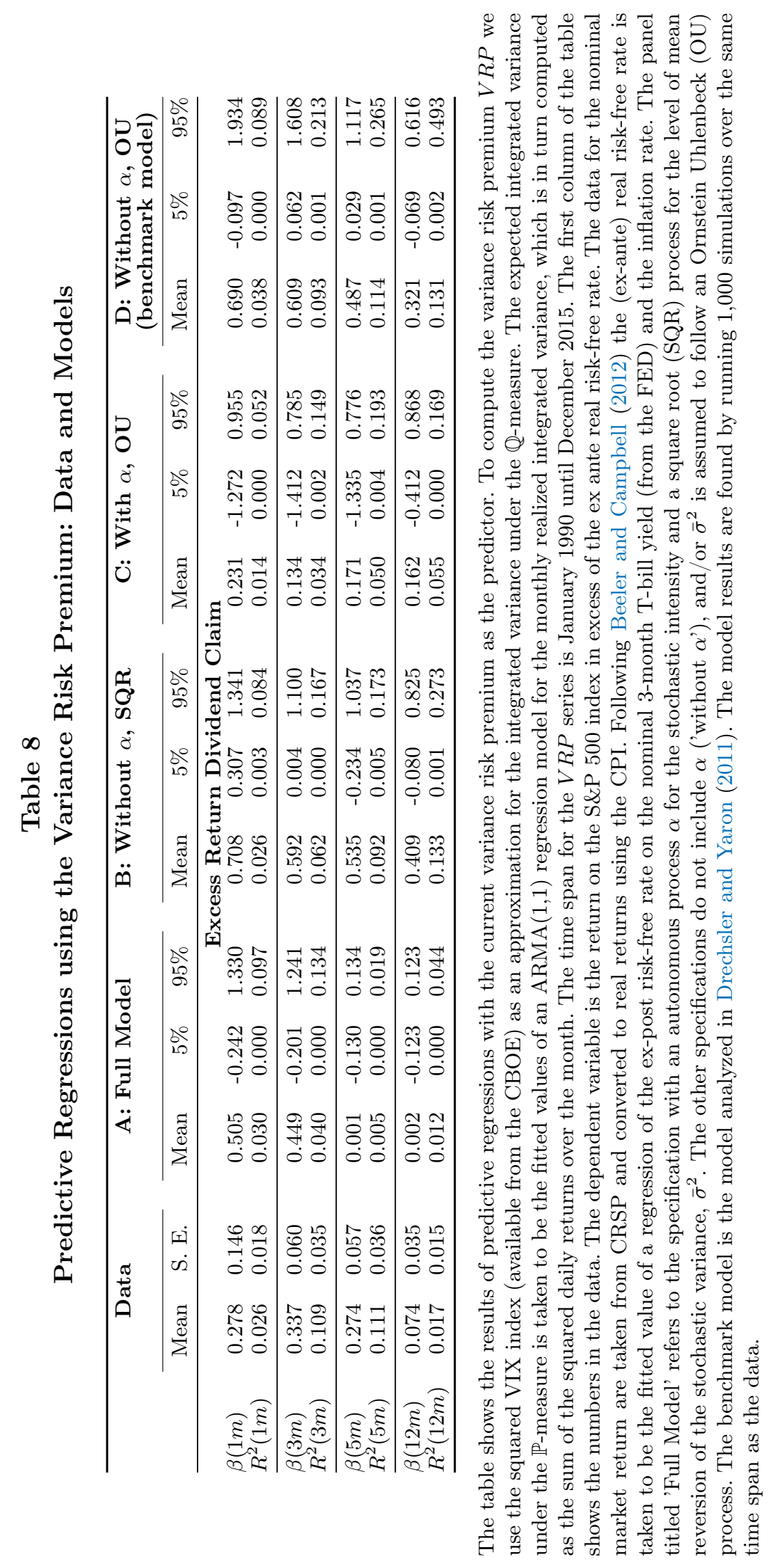




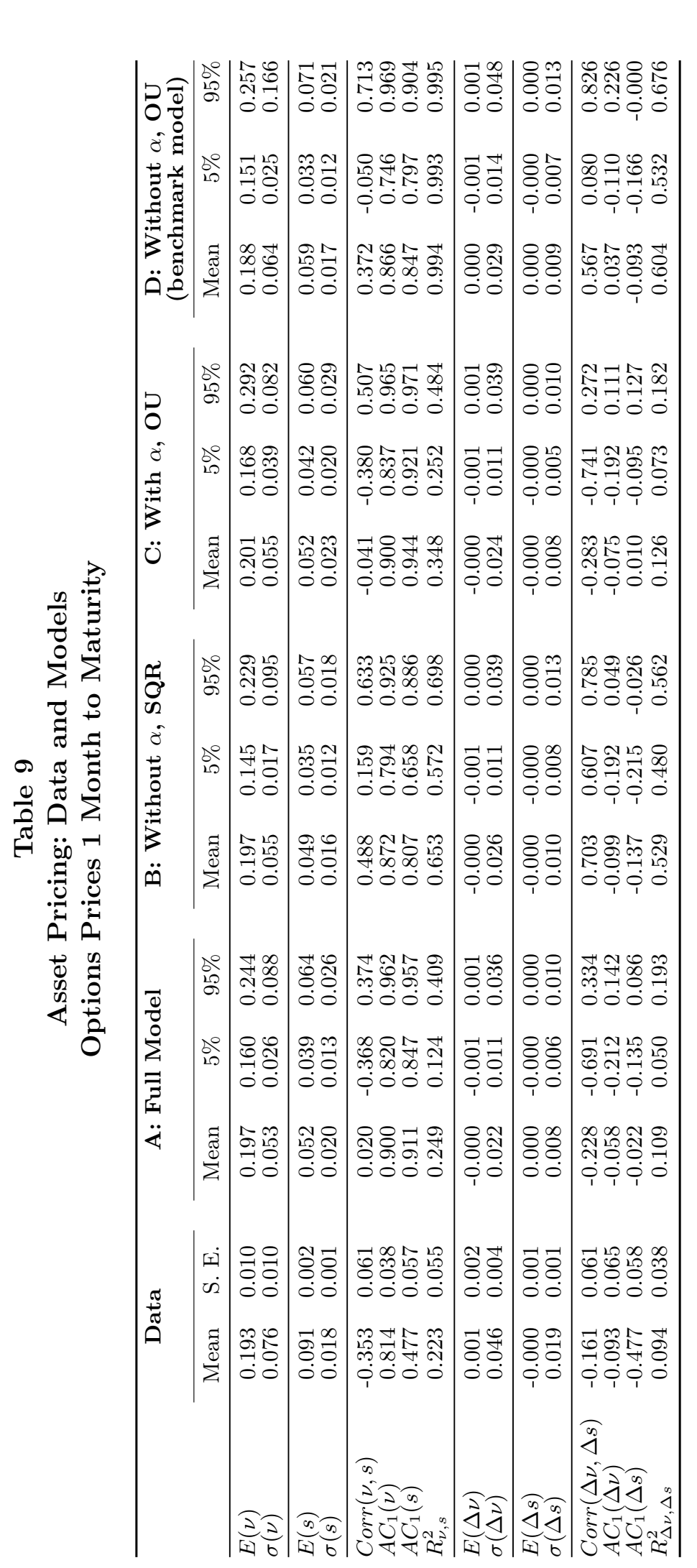

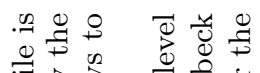

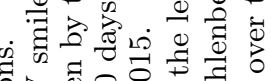

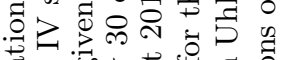
苞 F $\$$.

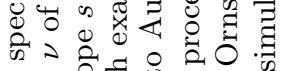

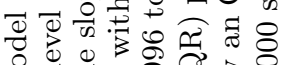

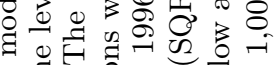

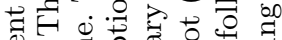
ज्ञ

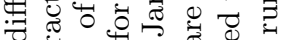
苛.

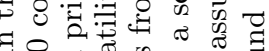
$\exists 8$ 중 สี 类需 (ن) Ð

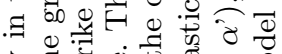

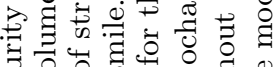
䙲

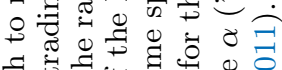

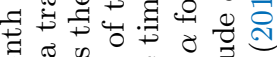

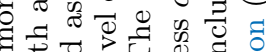

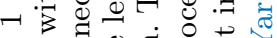

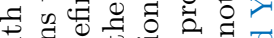
उ.

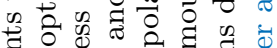

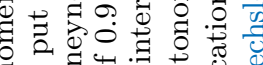
过 뭉 ๑ ॠ

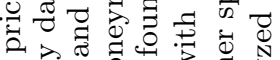

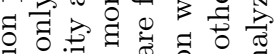
\%

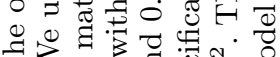
ॠ क्ष

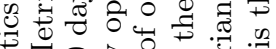

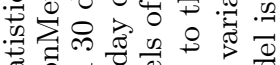

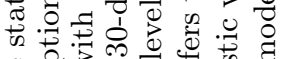

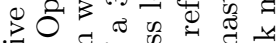

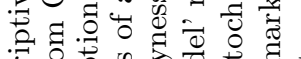

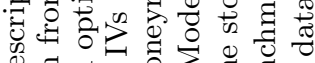

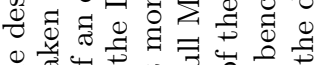

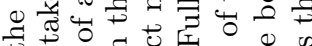

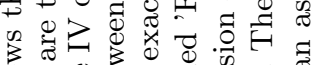

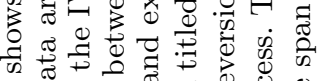

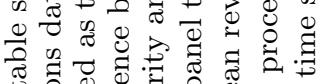

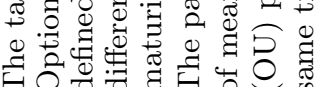




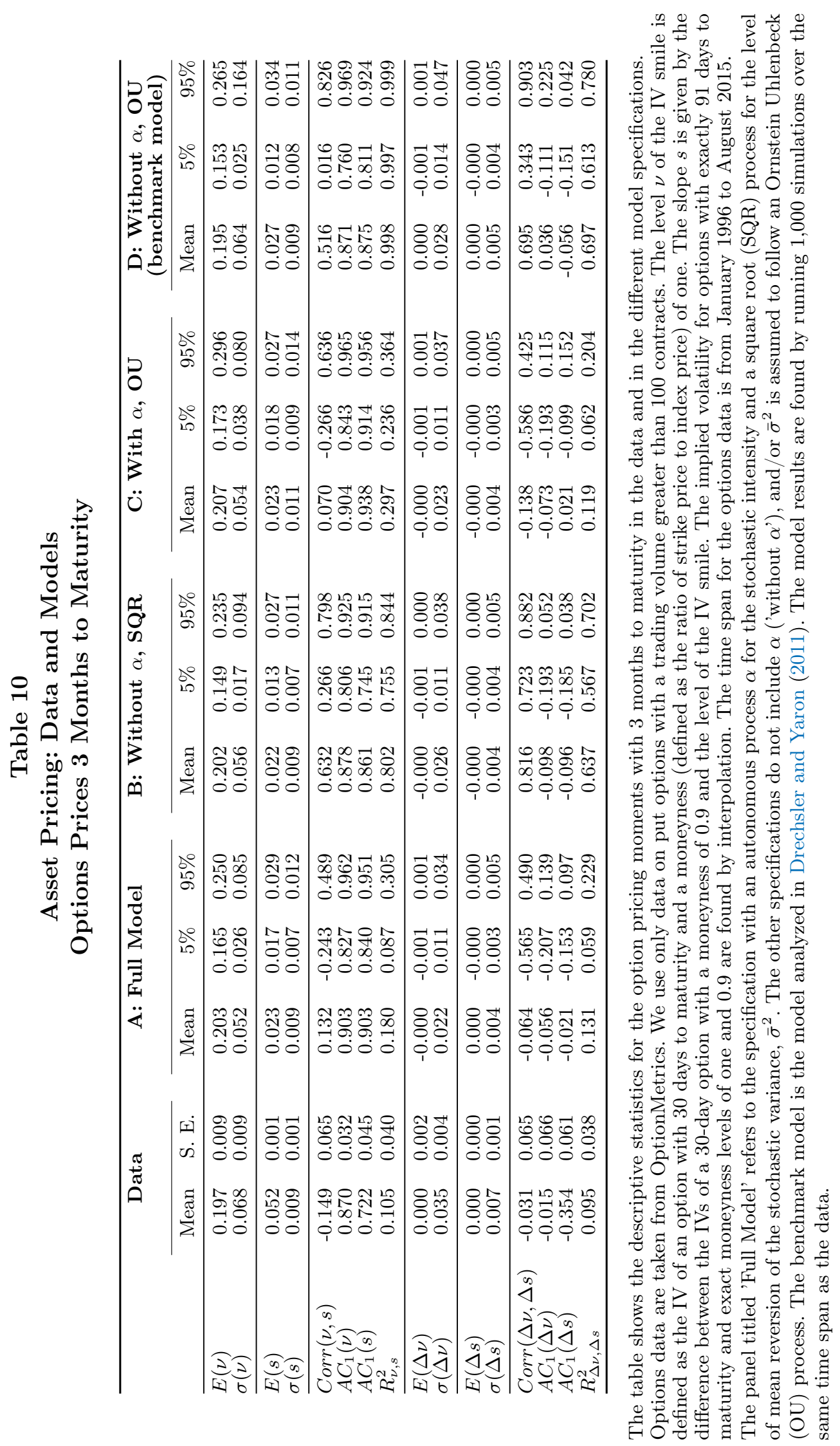




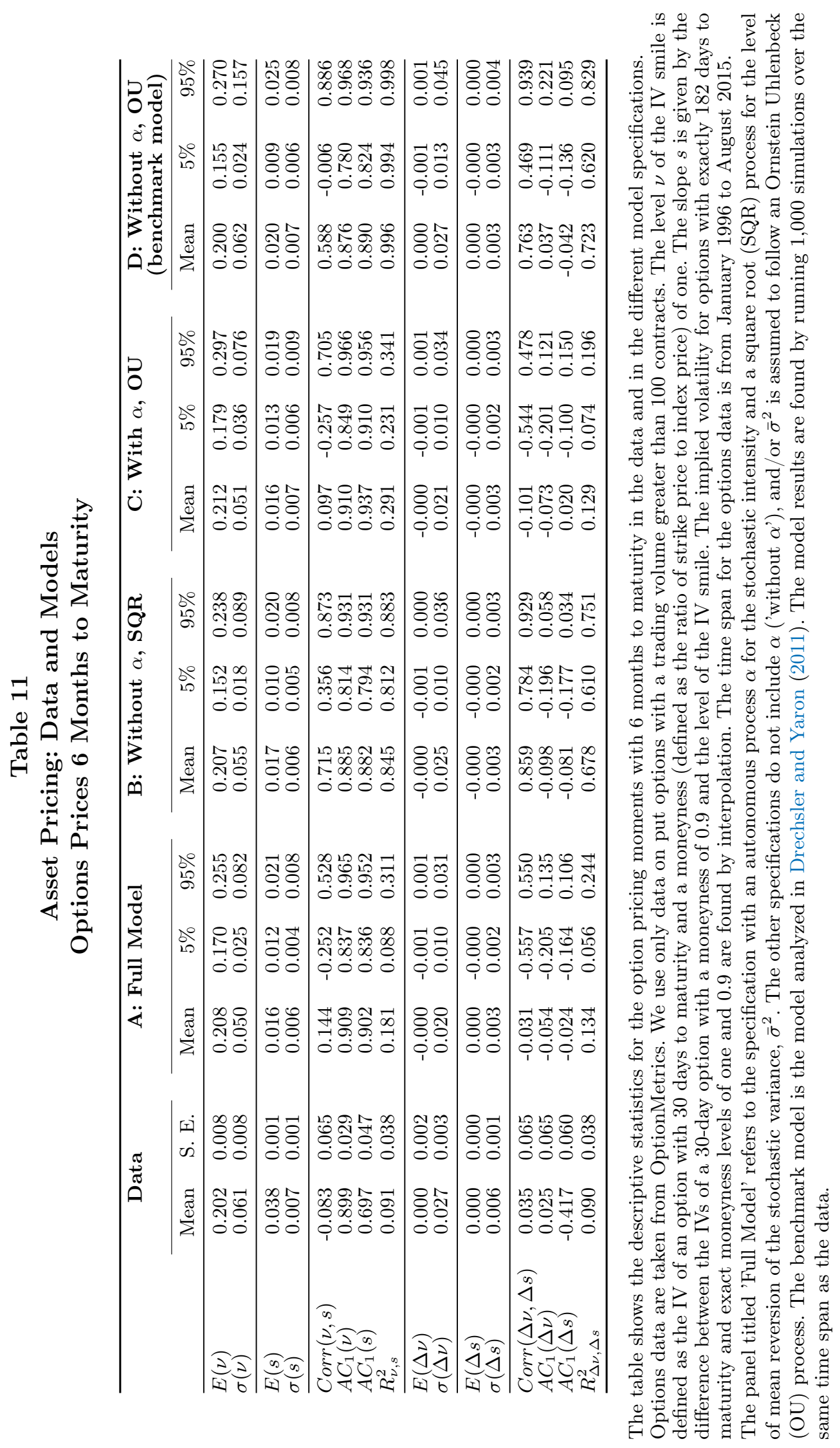




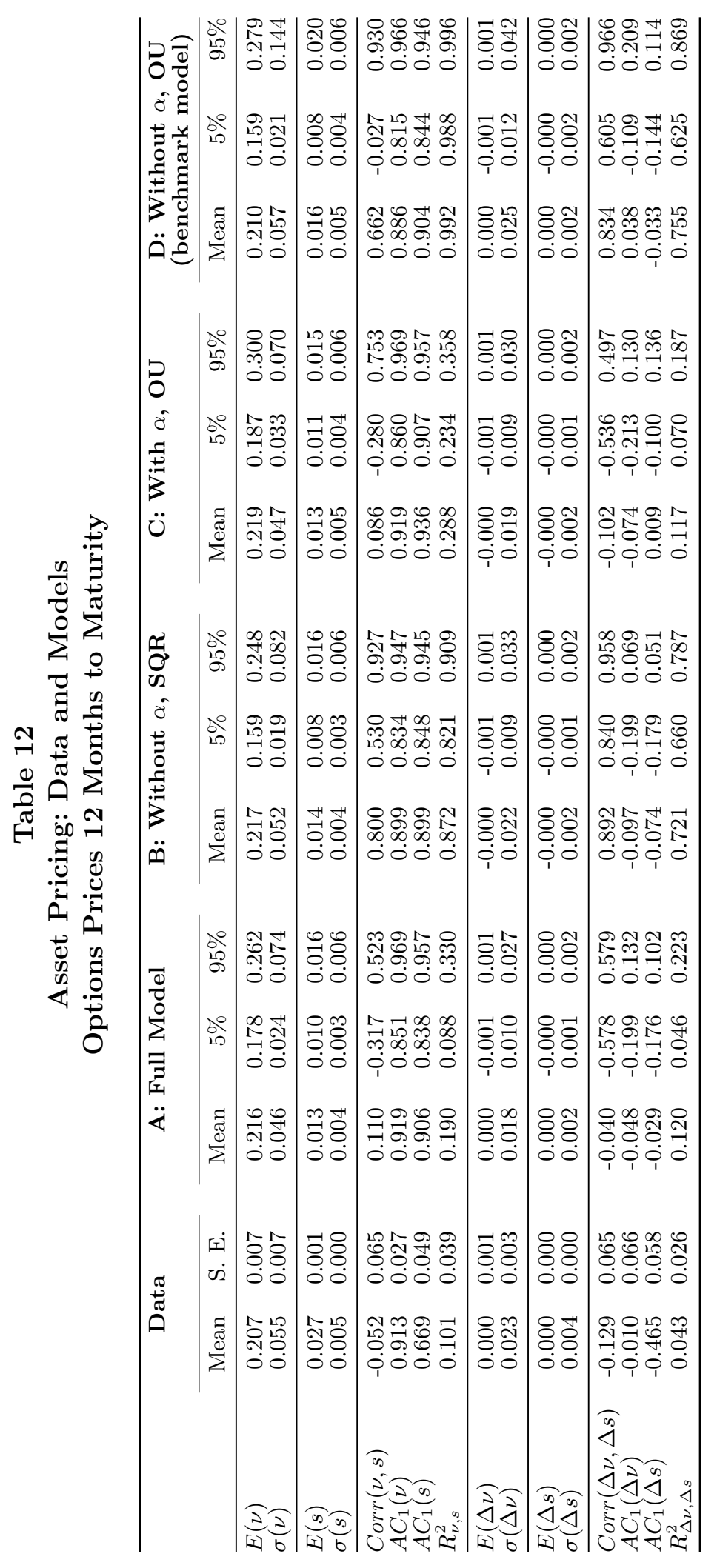

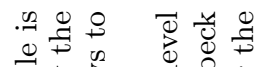

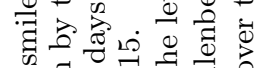

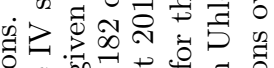

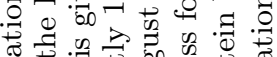
递

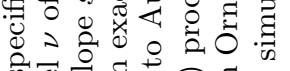
की

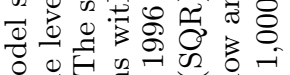
घ $\dot{F}$.

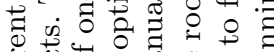

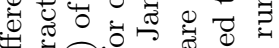
氙过

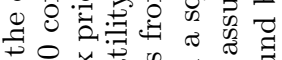

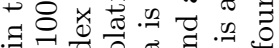

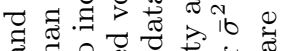

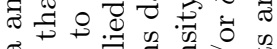

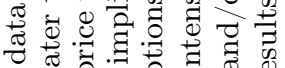

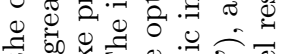
की घ द्वे

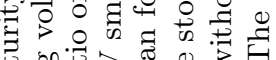

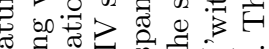

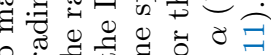

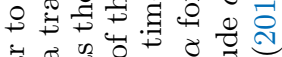

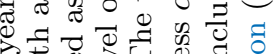
- 3 光

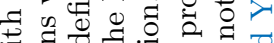
3.0 为

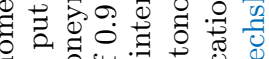

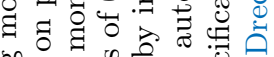

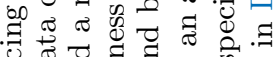

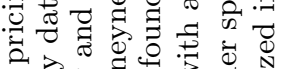

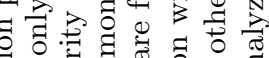
䒘药

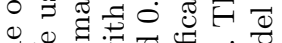

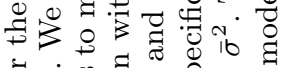
ड़े

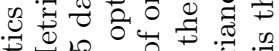

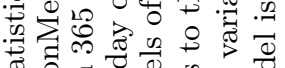

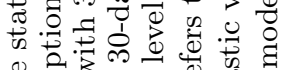
. वी 300

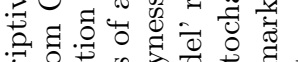

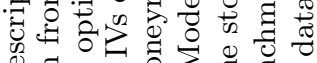
ชี ซี

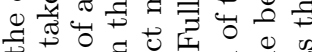

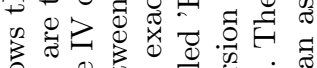

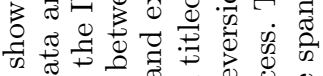

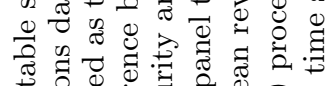

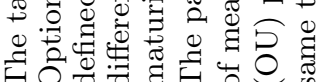


Table 13

Expected Excess Return on the Dividend Claim: Jump and Diffusive Risks

\begin{tabular}{|c|c|c|c|c|c|c|}
\hline & $x$ & $\sigma^{2}$ & $\alpha$ & $\bar{\sigma}^{2}$ & $\delta$ & Sum \\
\hline \multicolumn{7}{|c|}{ A: Full Model } \\
\hline Diffus & 1.125 & 0.156 & 0.003 & 1.039 & -0.371 & 1.952 \\
\hline Jump & 1.023 & 2.529 & 0.574 & - & 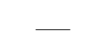 & 4.126 \\
\hline um & 2.147 & 2.685 & 0.577 & 1.039 & -0.371 & 6.078 \\
\hline \multicolumn{7}{|c|}{ D: Without $\alpha, \mathbf{S Q R}$} \\
\hline if & 1.281 & 0.243 & -0.000 & 2.106 & -0.306 & 3.324 \\
\hline & 1. & 2.2 & - & - & - & 3.443 \\
\hline sun & 2.457 & 2.510 & -0.000 & 2.106 & -0.306 & 6.767 \\
\hline \multicolumn{7}{|c|}{ C: With $\alpha$, OU } \\
\hline Diffu: & 1.131 & 0.158 & 0.003 & 0.767 & -0.371 & 1.689 \\
\hline Jum & 1.029 & 2.565 & 0.5 & - & 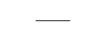 & 4.178 \\
\hline Sum & 2.160 & 2.723 & 0.587 & 0.767 & -0.371 & 5.867 \\
\hline \multicolumn{7}{|c|}{ B: Without $\alpha$, OU (benchmark model) } \\
\hline Ris & 1.307 & 0.256 & -0.000 & 1.191 & -0.306 & 2.449 \\
\hline Jump & 1.200 & 2.401 & - & - & - & 3.601 \\
\hline Sum & 2.507 & 2.657 & -0.000 & 1.191 & -0.306 & 6.050 \\
\hline
\end{tabular}

The table shows the decomposition of the expected excess returns on the dividend claim. All numbers are expressed in percentage points. They show which part of the total equity premium in each of the four versions of the model is due to diffusive risk in $x, \sigma^{2}, \alpha$, and $\bar{\sigma}^{2}$ and due to jump risk in $x, \sigma^{2}$, and $\alpha$. For example, the number ' 1.125 ' in the row 'Diffusive Risk' and the column labeled $x$ in Panel A means that 1.125 percentage points out of the total equity risk premium of $6.078 \%$ in this model are due to the fact that $\mathrm{x}$ exhibits variation driven by a diffusion process. Log dividends $\delta$ and the mean reversion level of volatility $\bar{\sigma}^{2}$ are purely diffusive processes without a jump component. The entries in the column labeled 'Sum' represent the respective sum of the diffusive and jump-related parts, evaluated at the long-run mean of the state variables, which is equal to 1 for $\sigma^{2}, \alpha$, and $\bar{\sigma}^{2}$. 
Table 14

Expected Excess Returns on the Dividend Claim: Impct of Current Values of the State Variables

\begin{tabular}{rrrrrr}
\hline \multicolumn{7}{c}{ Constant } & $\sigma^{2}$ & $\alpha$ & $\bar{\sigma}^{2}$ & Sum \\
\hline \multicolumn{7}{c}{ A: Full Model } \\
Diffusive Risk & -0.396 & 1.306 & 0.003 & 1.039 & 1.952 \\
Jump Risk & 0.000 & 0.000 & 4.126 & 0.000 & 4.126 \\
Sum & -0.396 & 1.306 & 4.129 & 1.039 & 6.078 \\
\hline \multicolumn{7}{c}{ D: Without $\alpha$, SQR } \\
Diffusive Risk & -0.349 & 1.566 & - & 2.106 & 3.324 \\
Jump Risk & 0.000 & 3.443 & - & 0.000 & 3.443 \\
Sum & -0.349 & 5.009 & - & 2.106 & 6.767 \\
\hline \multicolumn{7}{c}{ C: With $\alpha$, OU } \\
Diffusive Risk & 0.371 & 1.315 & 0.003 & 0.000 & 1.689 \\
Jump Risk & 0.000 & 0.000 & 4.178 & 0.000 & 4.178 \\
Sum & 0.371 & 1.315 & 4.181 & 0.000 & 5.867 \\
\hline B: Without $\alpha$, OU (benchmark model) \\
Diffusive Risk & 0.843 & 1.606 & - & 0.000 & 2.449 \\
Jump Risk & 0.000 & 3.601 & - & 0.000 & 3.601 \\
Sum & 0.843 & 5.208 & - & 0.000 & 6.050 \\
\hline \multicolumn{7}{c}{}
\end{tabular}

The table shows the decomposition of the diffusive and the jump-driven part of the expected excess returns on the dividend claim into a constant and terms which are proportional to the state variables $\sigma^{2}$, $\alpha$, and $\bar{\sigma}^{2}$. For example, the entries in the row 'Diffusive Risk' in Panel A mean that the diffusive part of the equity risk premium in the full model can be written as $-0.396+1.306 \sigma^{2}+0.003 \alpha+1.039 \bar{\sigma}^{2}$. The entries in the column labeled 'Sum' represent the respective sum of the diffusive and jump-related parts, evaluated at the long-run mean of the state variables, which is equal to 1 for $\sigma^{2}, \alpha$, and $\bar{\sigma}^{2}$. All numbers are expressed in percentage points. 


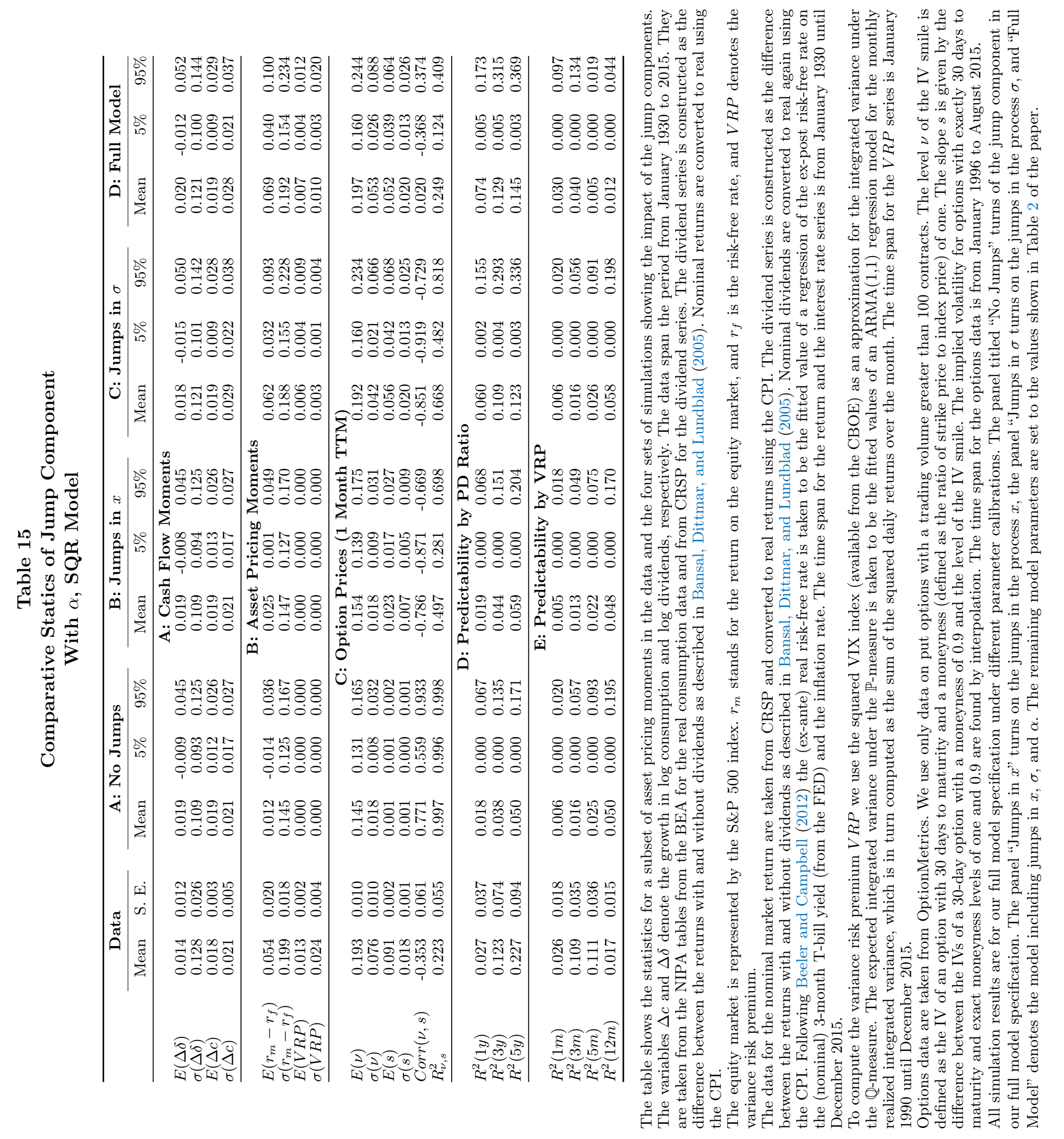




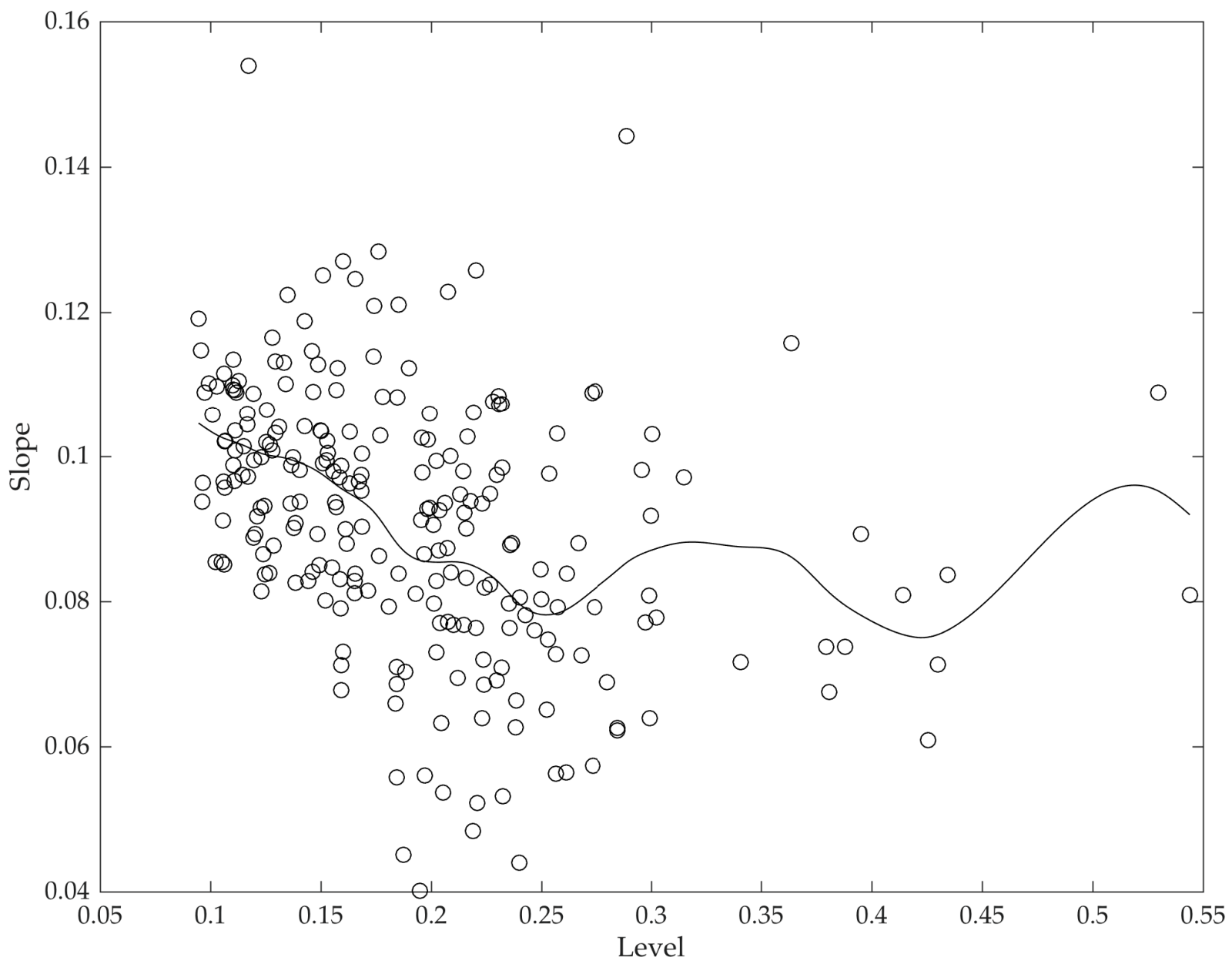

Figure 1: Level and Slope of the S\&P 500 Implied Volatility Smile

The figure shows the end-of-the-month level and slope of the implied volatility smile for S\&P 500 options with 30 days to maturity. The level is defined as the implied volatility of a put option with moneyness (strike price divided by current index level) equal to 1 . The slope is defined as the difference between the implied volatilities of two put options with moneyness equal to 0.9 and 1, respectively. The time period spans from January 1996 until December 2015. 

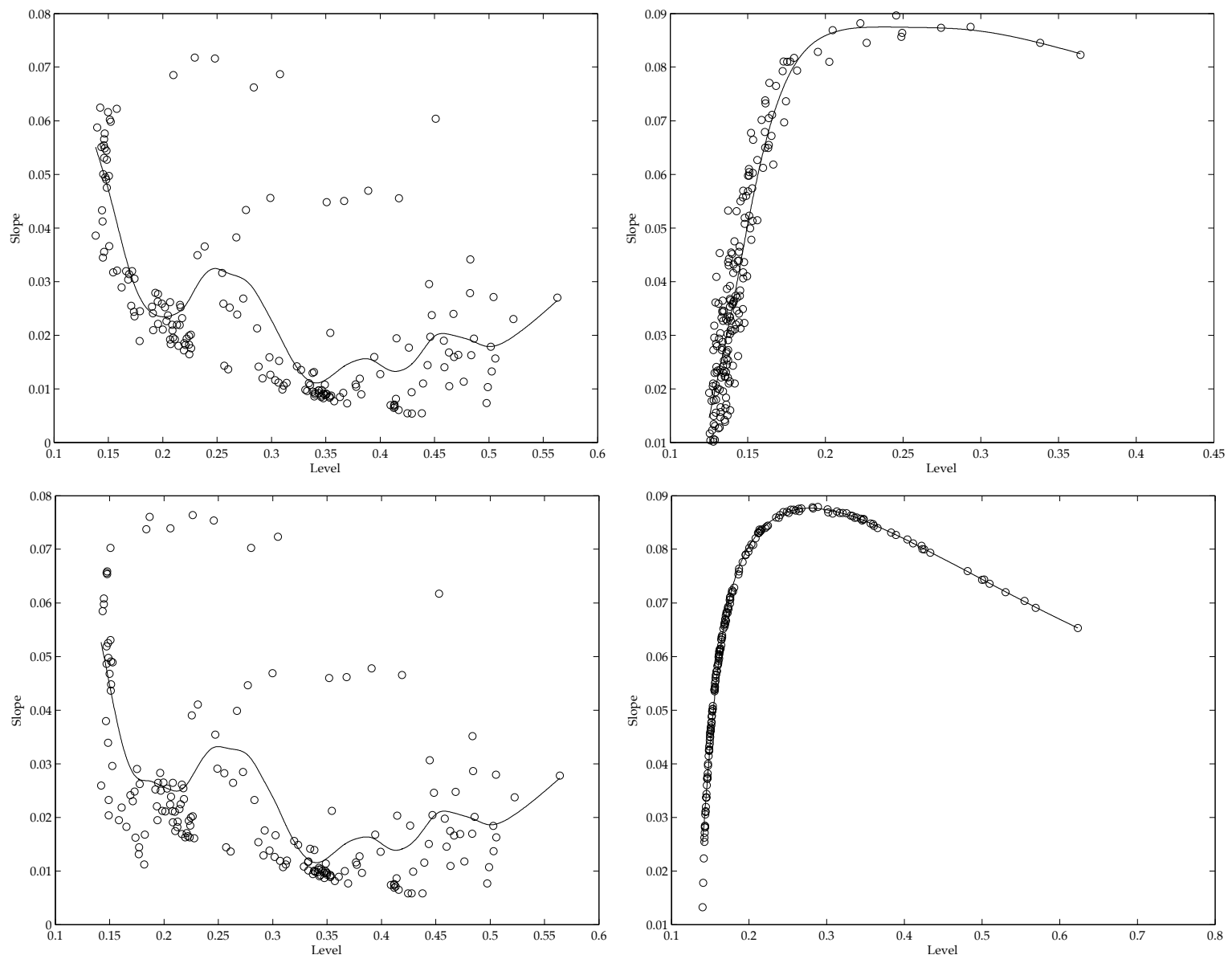

Figure 2: Level and Slope Implied Volatility Smile in Different LRR-Models

The figure shows the model-generated level and the slope of the implied volatility smile for options with 1 month to maturity. The level is defined as the implied volatility of a put option with moneyness (strike price divided by current index level) equal to 1 . The slope is defined as the difference between the implied volatilities of two put options with moneyness equal to 0.9 and 1 , respectively. The upper left graph is for our model with a separate intensity process and a square-root specification for the long-run mean of the conditional variance. The upper right graph represents the model with intensity affine in variance and a square-root process for the long-run mean, while the lower left picture is for the specification with separate processes for intensity and variance, but with an Ornstein-Uhlenbeck process for the long-run mean. Finally, the lower right graph is for the model where intensity is affine in the variance and the long-run mean follows an Ornstein-Uhlenbeck process. 


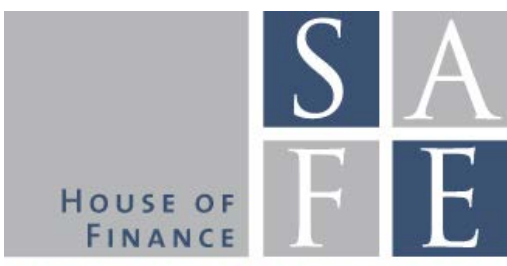

WORKING PAPER SERIES

\section{Recent Issues}

No. 185 Patrick Grüning

No. 184 Tobias Tröger

No. 183 Joost Driessen, Theo E. Nijman, Zorka Simon

No. 182 Mario Bellia, Loriana Pelizzon, Marti G. Subrahmanyam, Jun Uno, Darya Yuferova

No. 181 Holger Kraft, Farina Weiss

No. 180 Tobias H. Tröger

No. 179 Tobias H. Tröger

No. 178 Matthias Goldmann

No. 177 Michael Donadelli, Marcus Jüppner, Max Riedel, Christian Schlag

No. 176 Giuliano Curatola, Ilya Dergunov

No. 175 Reint Gropp, Deyan Radev

No. 174 Reint Gropp, Deyan Radev
Heterogeneity in the Internationalization of R\&D: Implications for Anomalies in Finance and Macroeconomics

Remarks on the German Regulation of Crowdfunding

The Missing Piece of the Puzzle: Liquidity Premiums in Inflation-Indexed Markets

Coming Early to the Party

Consumption-Portfolio Choice with Preferences for Cash

Why MREL Won't Help Much

Too Complex to Work: A Critical Assessment of the Bail-in Tool under the European Bank Recovery and Resolution Regime

United in Diversity? The Relationship between Monetary Policy and Banking Supervision in the Banking Union

Temperature Shocks and Welfare Costs

International Capital Markets with TimeVarying Preferences

International Banking Conglomerates and the Transmission of Lending Shocks across Borders

Social Centralization, Bank Integration and the Transmission of Lending Shocks 